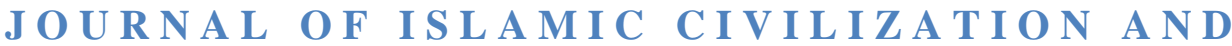
C U L T U R E ( J I C C )

Volume 3, Issue 1 (Jan-June, 2020)

ISSN (Print):2707-689X

ISSN (Online) 2707-6903

Issue: http://ahbabtrust.org/ojs/index.php/jicc/issue/view/8

URL: http://ahbabtrust.org/ojs/index.php/jicc/article/view/86/98

Article DOI: https://doi.org/10.46896/iicc.v3i01.86

Title

Research Review of the Tolerance of Muslims with Non-Muslims in Spain and its Impacts

Author (s): $\quad$ Rooh Ullah, Dr. Mushtaq Ahmad

Received on: 29 June, 2019

Accepted on: 29 May, 2020

Published on: 25 June, 2020

Citation: Rooh Ullah and Dr. Mushtaq Ahmad, "Construction: Research Review of the Tolerance of Muslims with Non-Muslims in Spain and its Impacts," JICC: 3 no, 1 (2020): 94-110

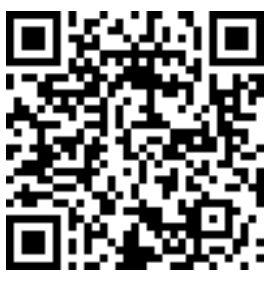

Scientific Indexing
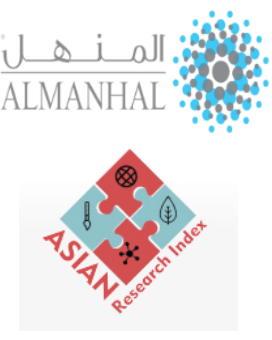

Crossref 


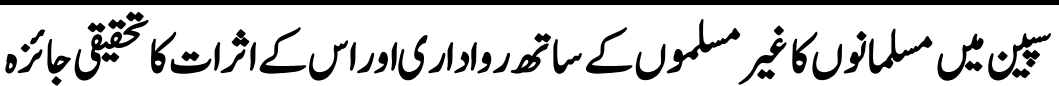

\section{Research Review of the Tolerance of Muslims with Non-Muslims in Spain and its Impacts}

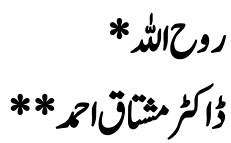

\section{Abstract:}

Islamic ideology is the basis and source of Islamic state, which sets out the rights of Muslims as well as the Dhimmis. Islam teaches the tolerance and fairness to nonMuslims citizens. Islam gives the non-Muslims religious freedom. Quran says, "There is no compulsion in Faith". Prophet Muhammad (P.B.U.H) says, "If anyone wrongs a Mu'ahid, detracts from his rights, burdens him with more work than he is able to do, or takes something from him without his consent, I will plead for him on the Day of Resurrection". Arab Muslims conquered Spain in 711 A.C. The Muslims defeated Christians there, while the Jews also existed there. When the Muslims (Moors) conquered this country, they behaved and treated the people here with fairness and tolerance. The tolerance of Muslims has had a profound impact on non-Muslims and the environment here. Many of non-Muslims converted to Islam with their own consent. Muslims gave them full enfranchise to worship according to their own religion; the priest did not need to hide their religious status. Muslim Spain had complete freedom of education which led to students coming from other countries for pursuit education. Non-Muslims adopted culture, living style and ways to dress of Muslims. They learned Arabic and began to read poetry in Arabic. Arabic literature translated into Hebrew and Latin by non-Muslims. In Muslim Spain there was freedom of expression. The Jewish scholar Ibn Naghrila spoke on the beliefs of Muslims under the Muslim rule in Spain.

Hasdai ibn Shaprot (d.970) established a madrasa for Jews in Cordova to teach the Holy Scripture and Talmud. Katie Magnus (d.1924) says, "Like a dream in the night Life in Spain". Due to the tolerance of Muslims, Europeans became aware of civilization and from that time renaissance began. Muslims behaved non-Muslims with tolerance, contrary to non-Muslims, while they overcome on Muslims, wherever their attitude with Muslims is always regrettable. With the fall of the Muslim's empire, Spain fell into the darkness of ignorance. Stanley lane-Poole (d.1931) says, "The Moors were banished, for a while the Christian Spain shone, like the Moon, with a borrowed light, then came the eclipse, and in that darkness Spain grovelled ever since".

Keywords: Spain, Muslims, Non-Muslims, Tolerance, Impacts.

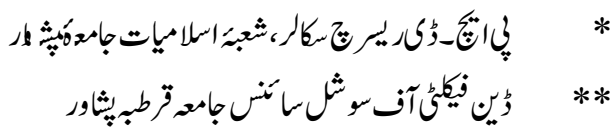




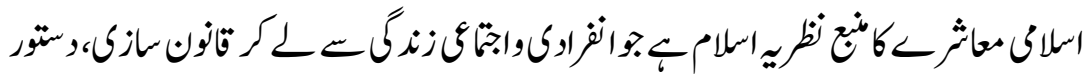

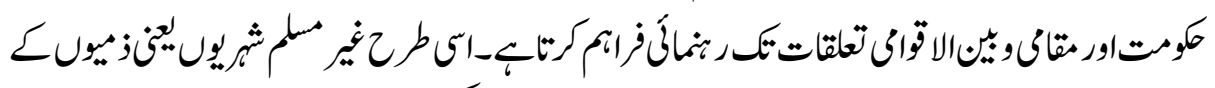

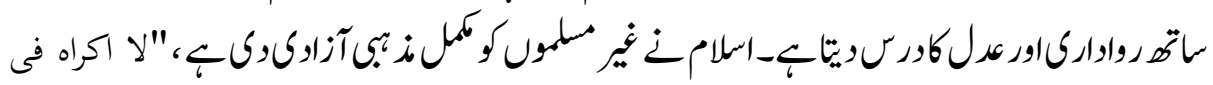

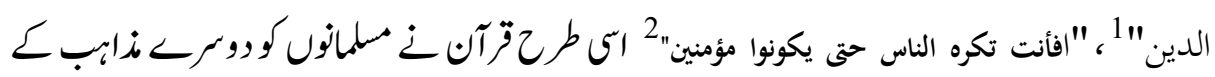

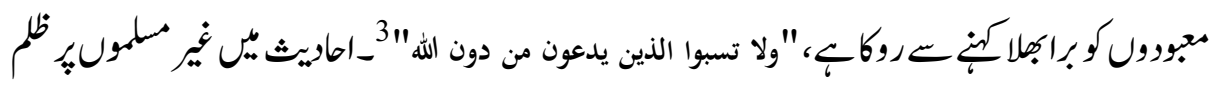

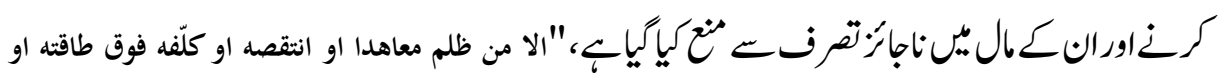

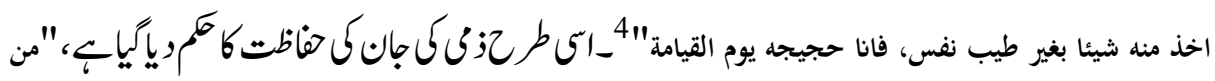

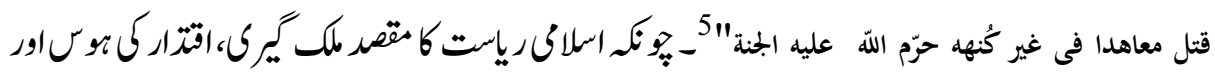

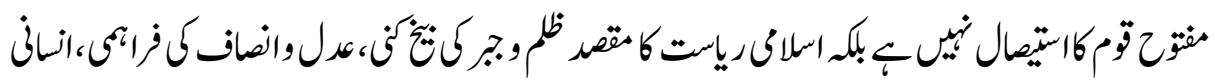

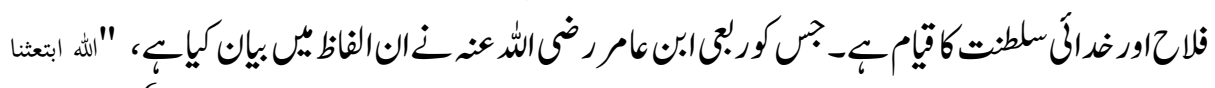

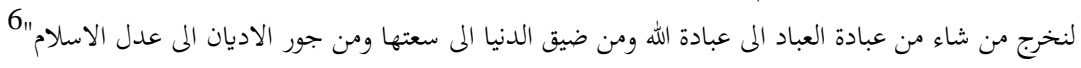

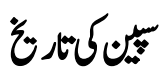

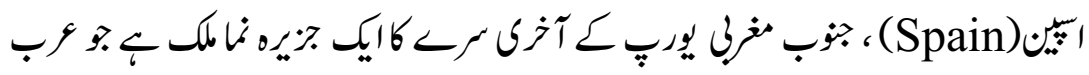

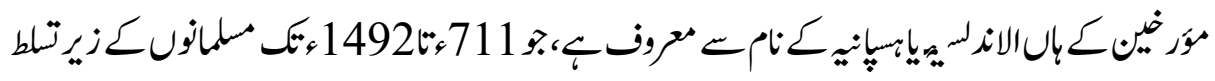

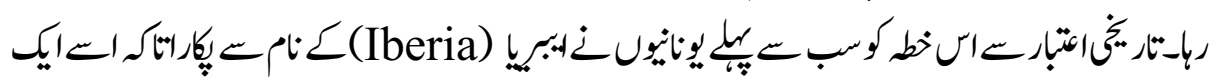

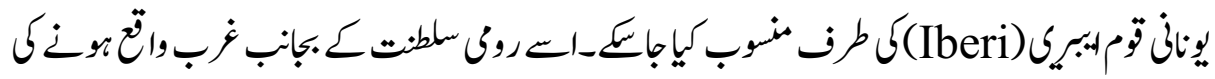

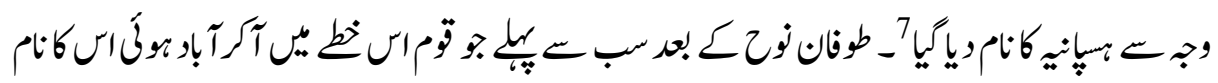

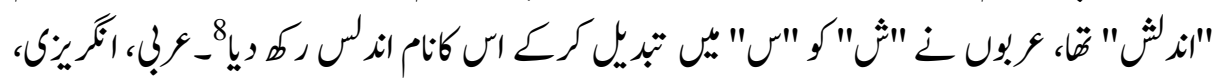

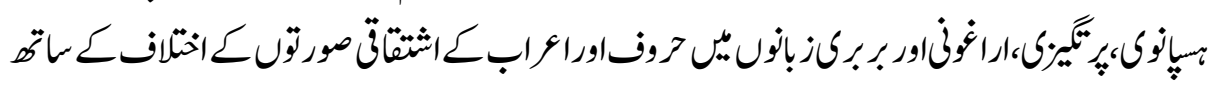

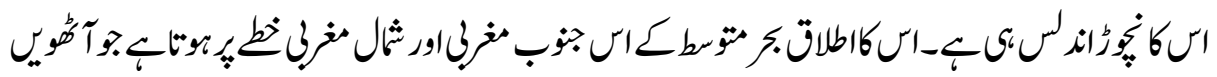

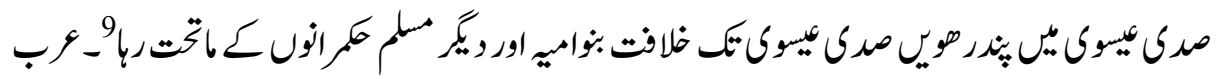

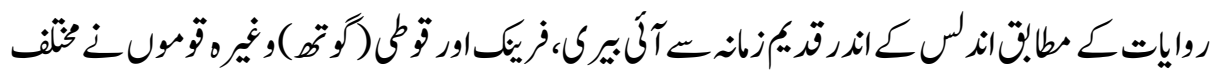

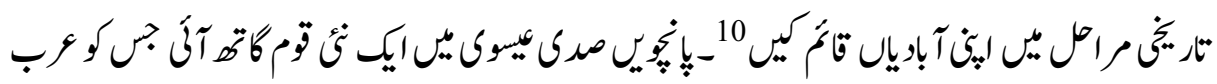

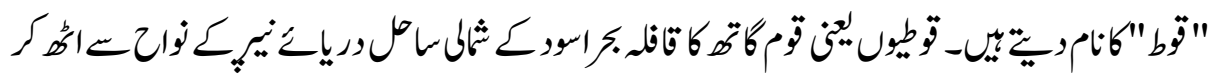




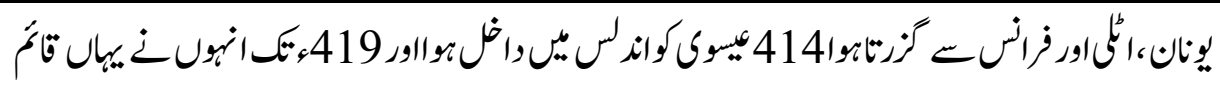

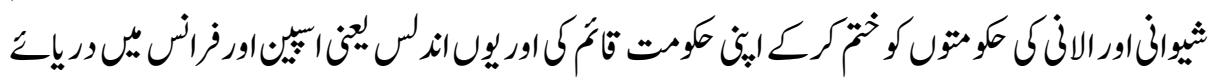

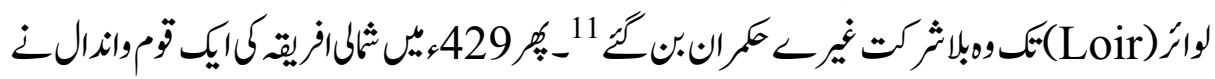

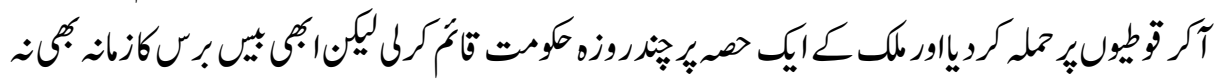

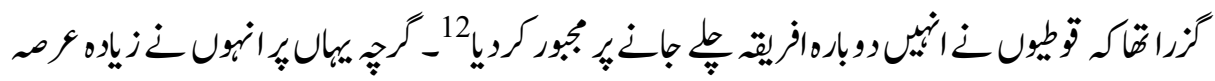

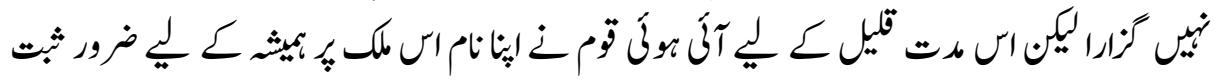

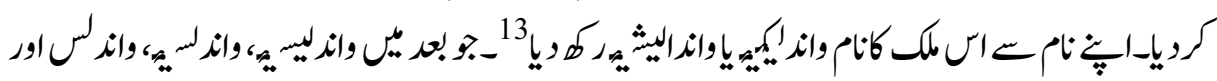

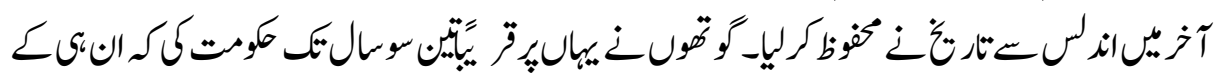

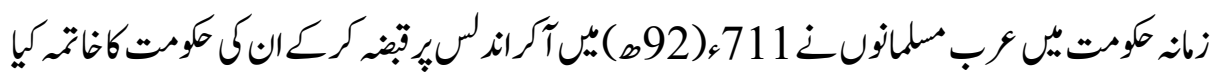

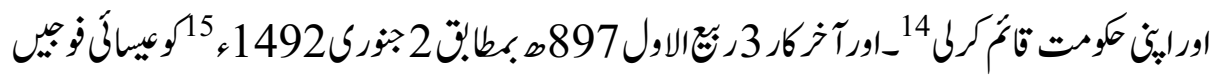

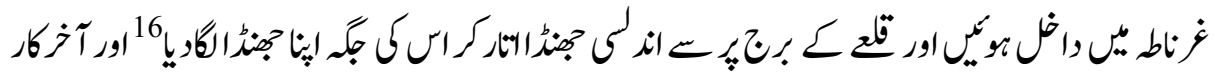

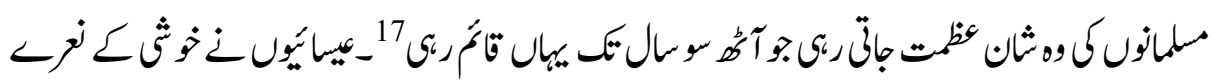

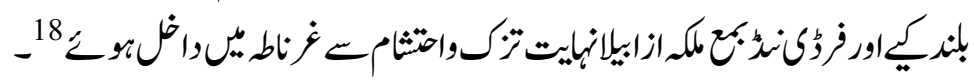

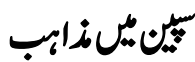

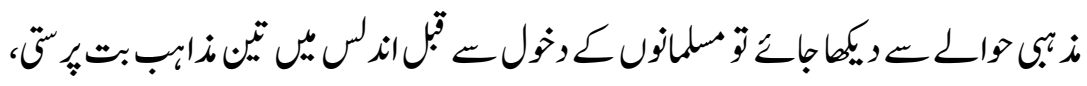

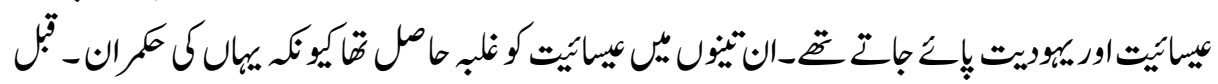

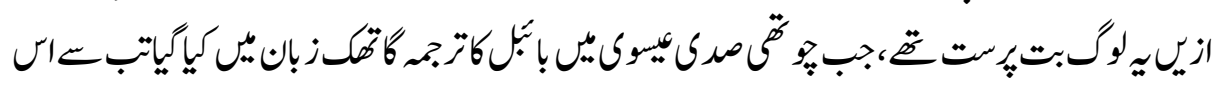

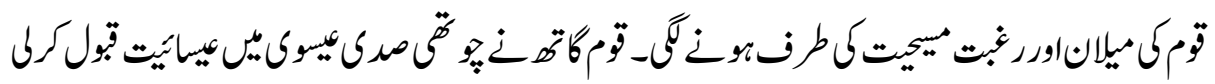

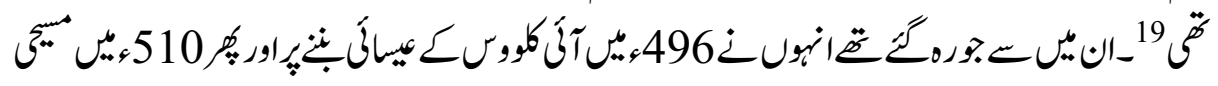

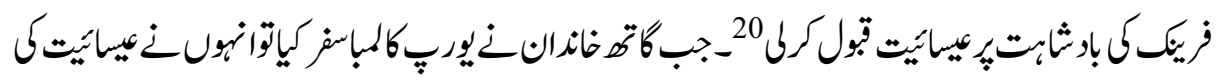

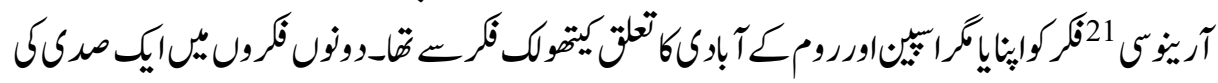

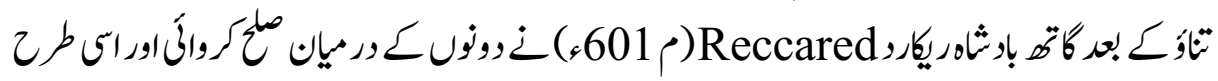

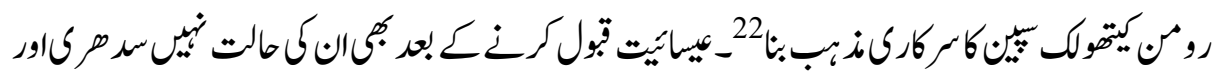

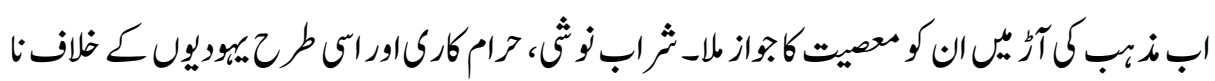




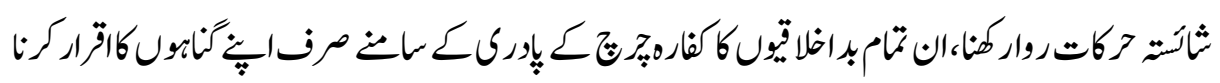

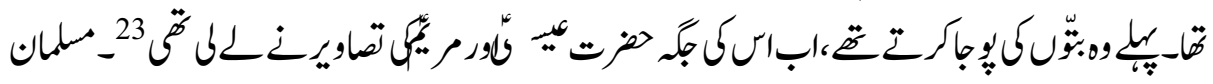

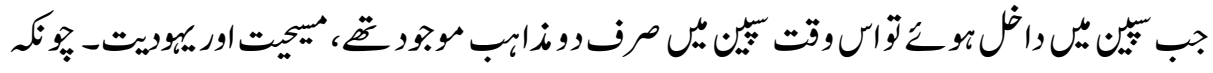

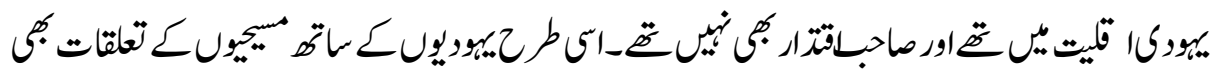

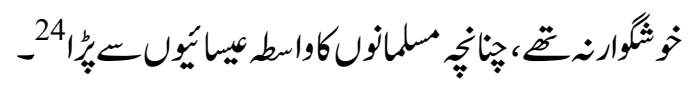

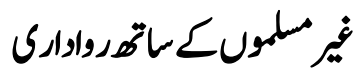

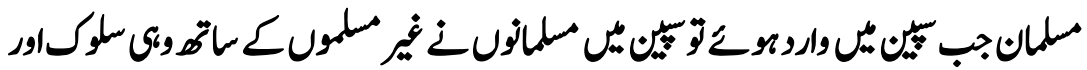

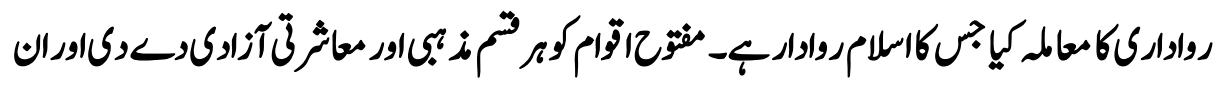

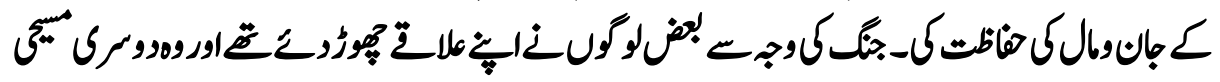

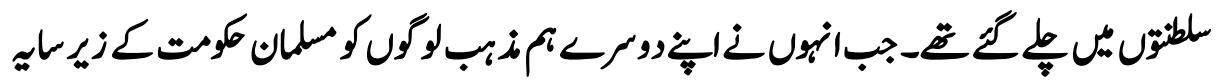

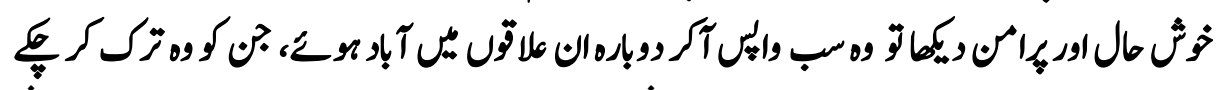

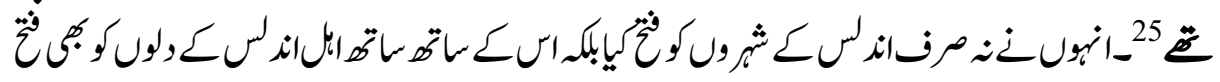

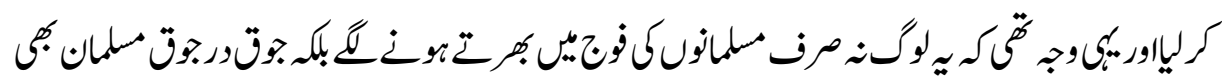

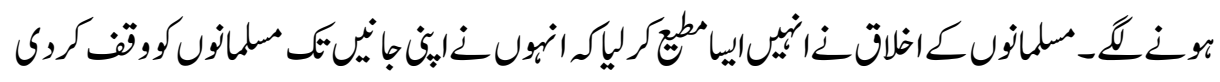

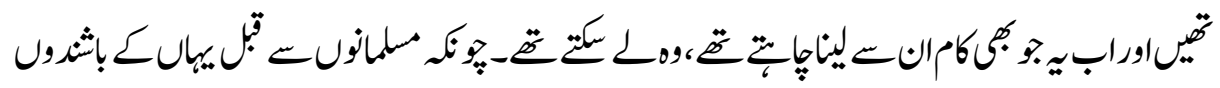

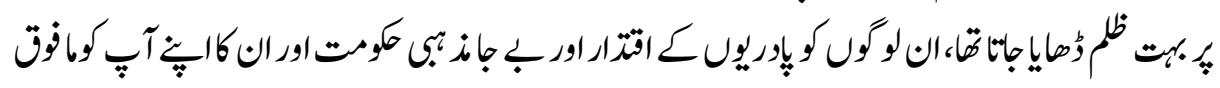

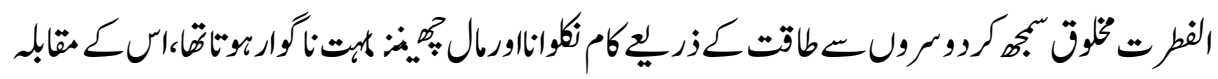

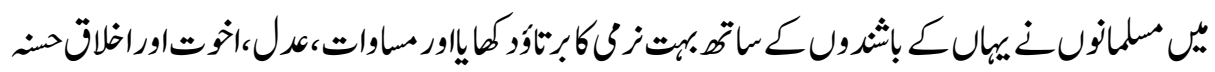

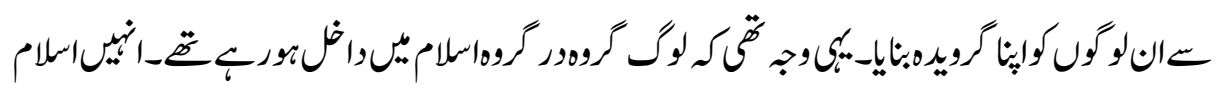

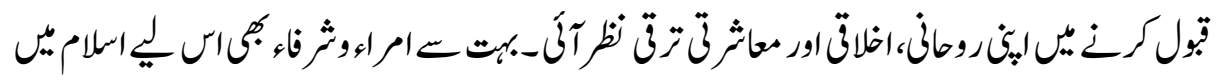

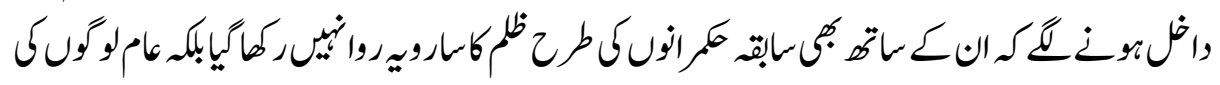

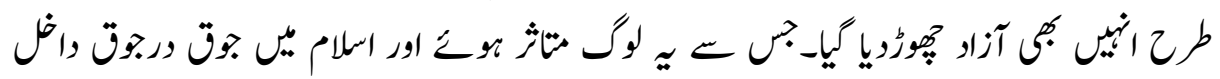

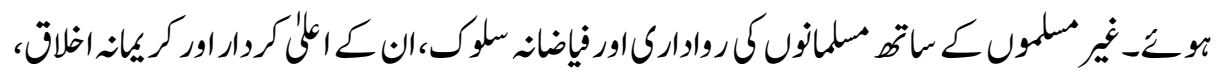

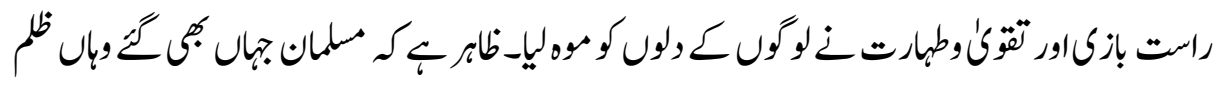




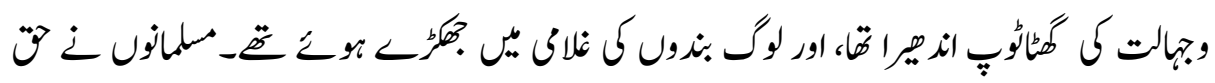

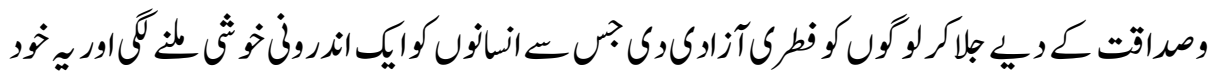

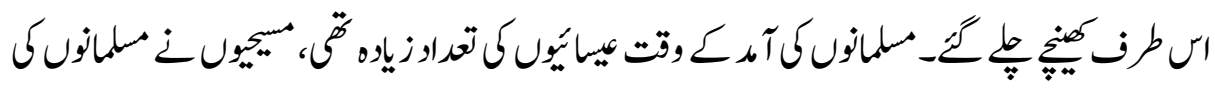

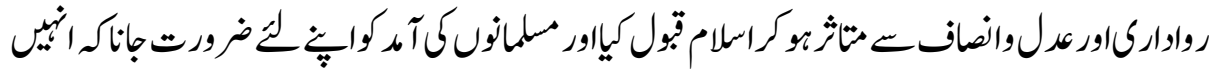

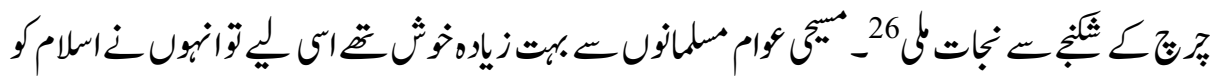

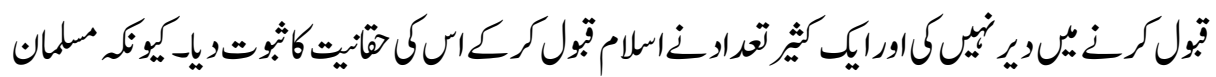

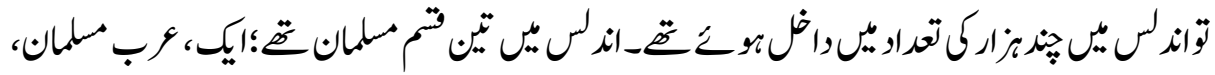

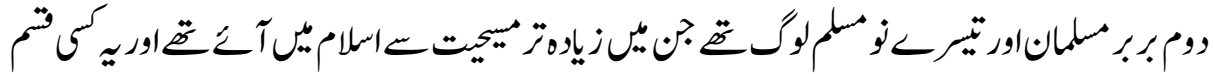

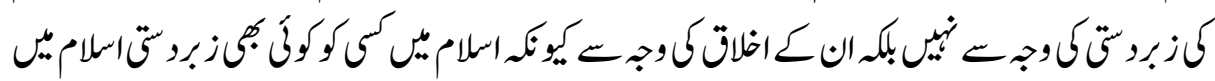

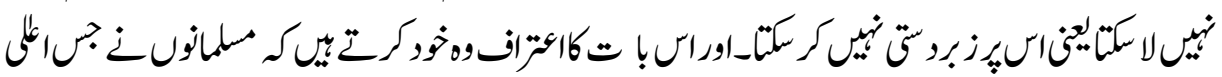

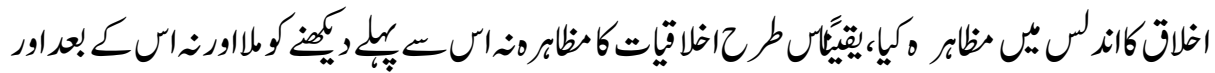

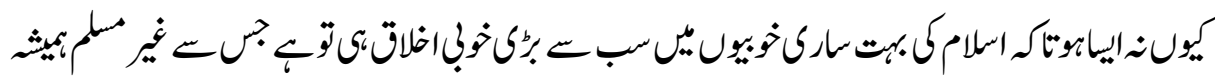

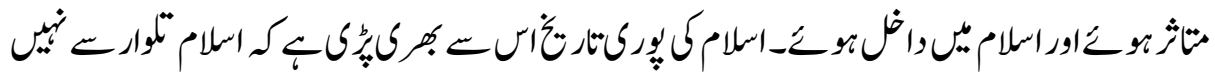

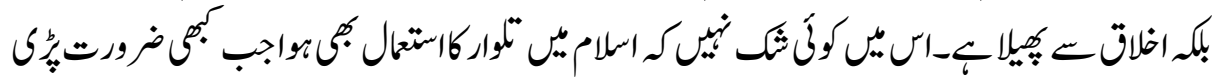

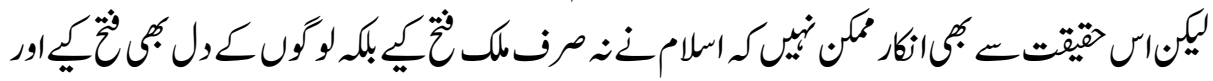

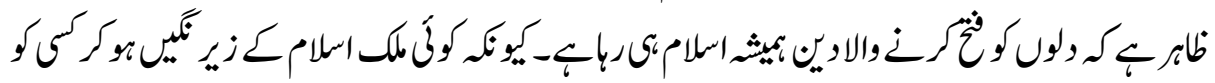

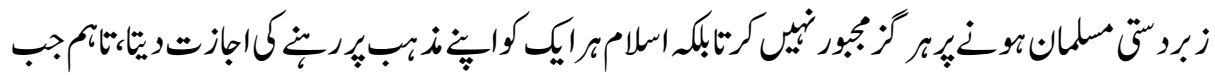

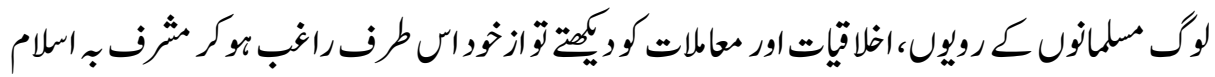

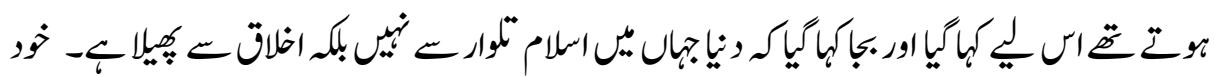

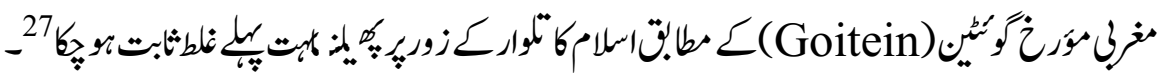

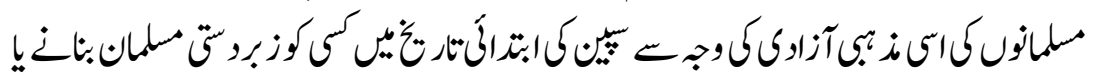

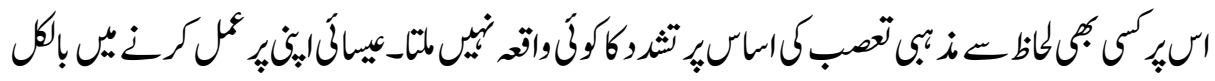

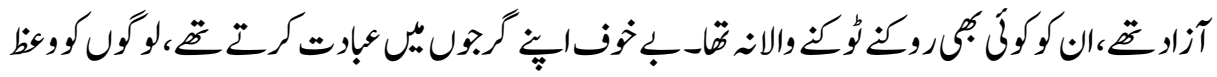

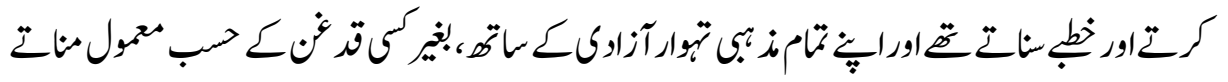




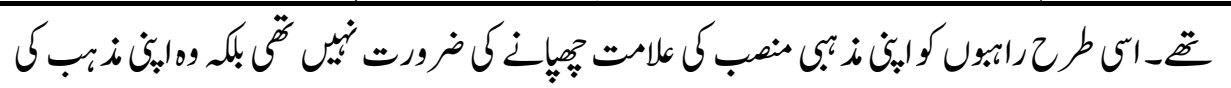

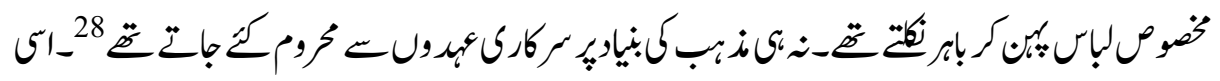

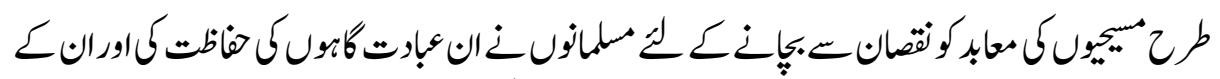

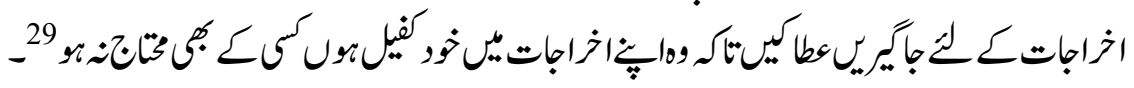

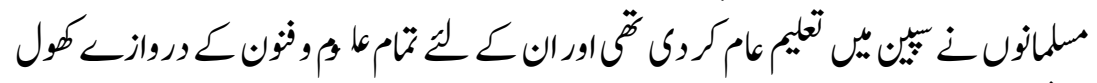

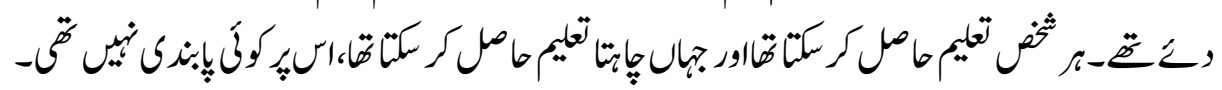

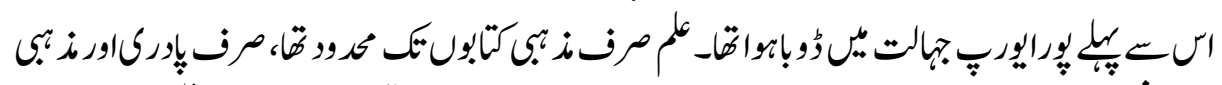

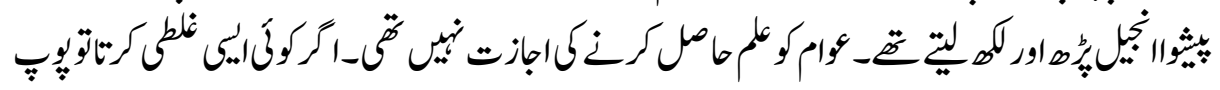

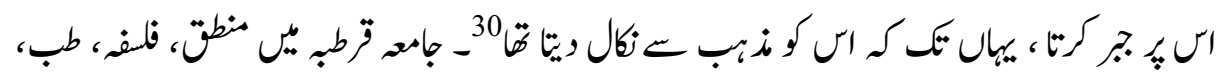

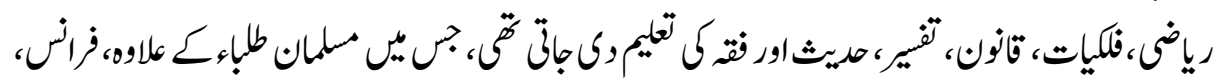

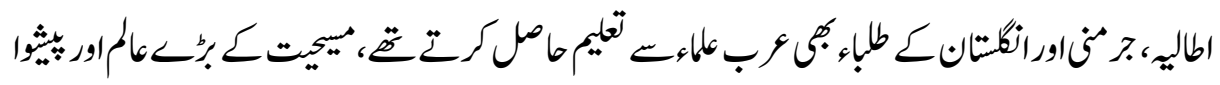

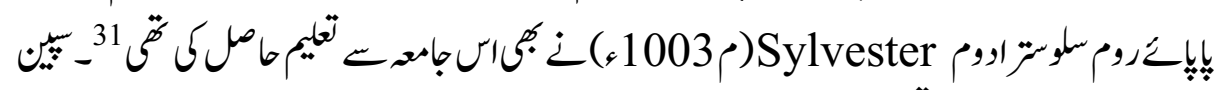

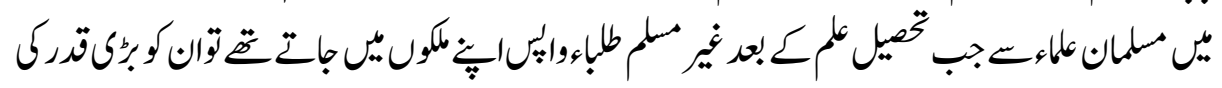

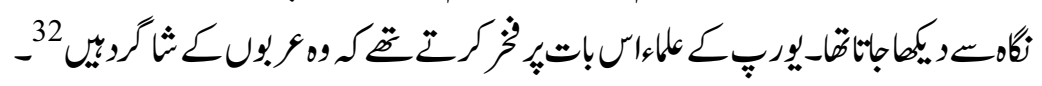

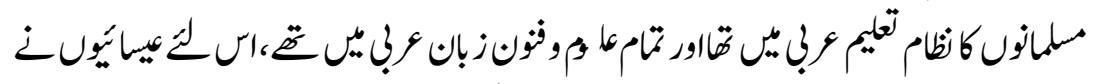

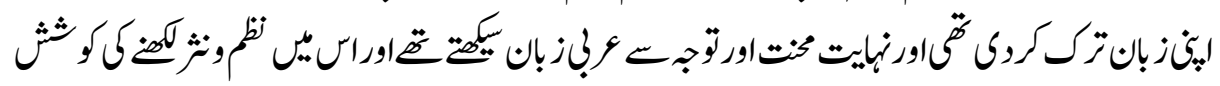

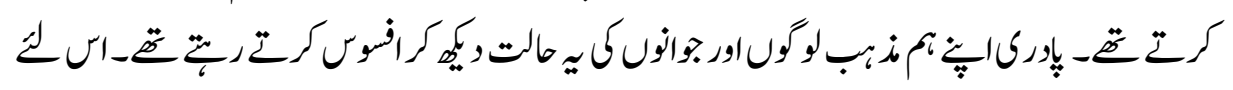

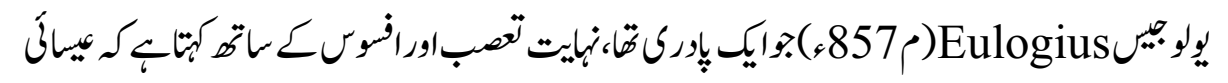

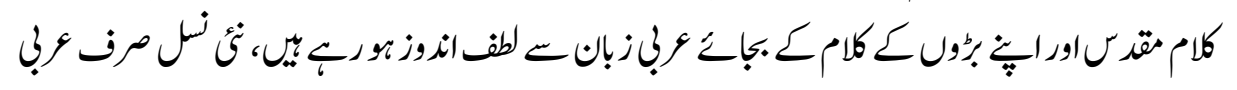

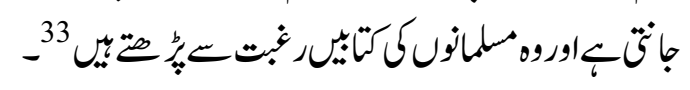

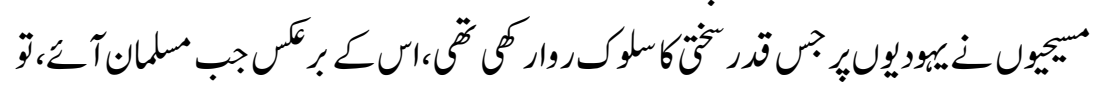

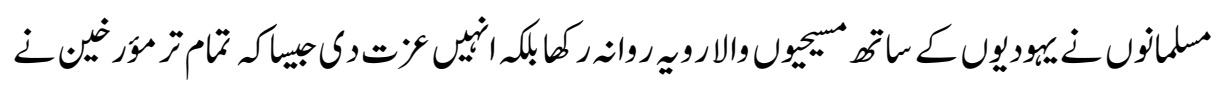

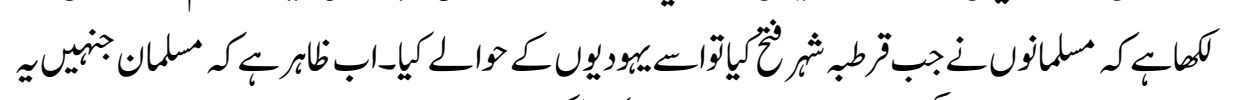

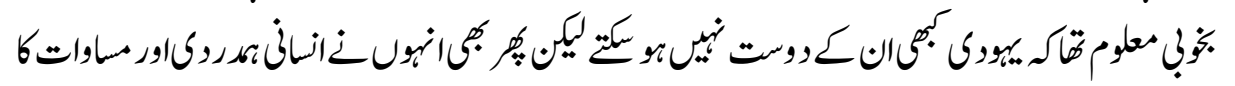




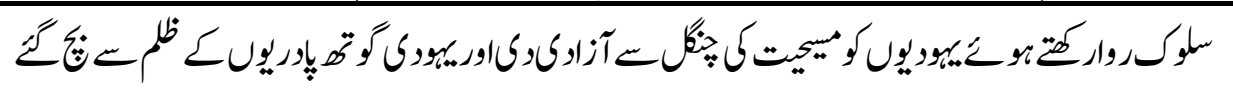

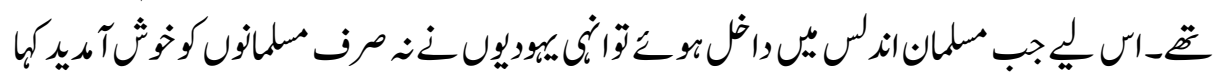

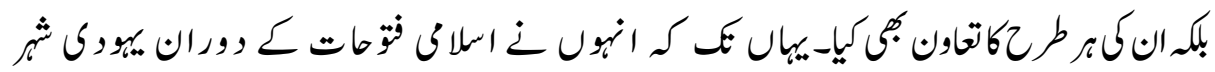

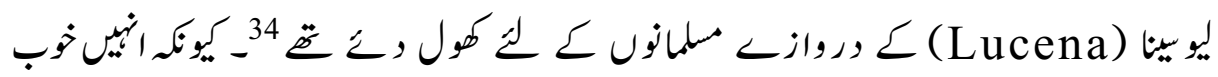

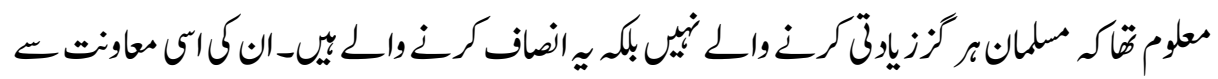

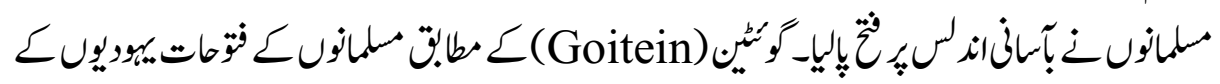

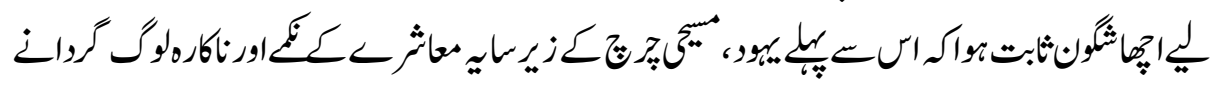

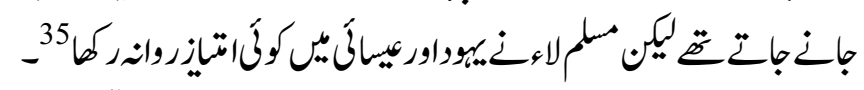

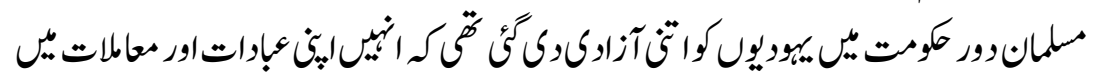

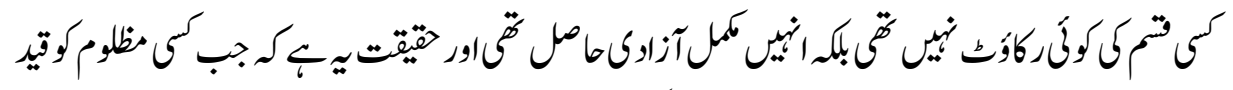

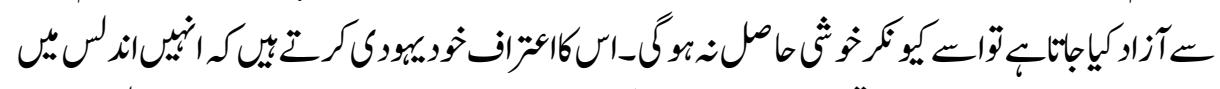

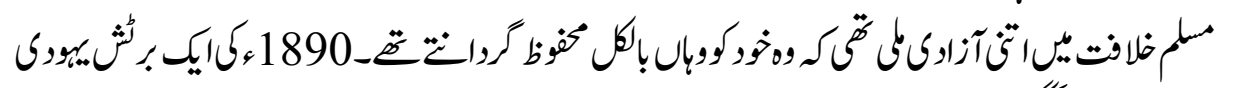

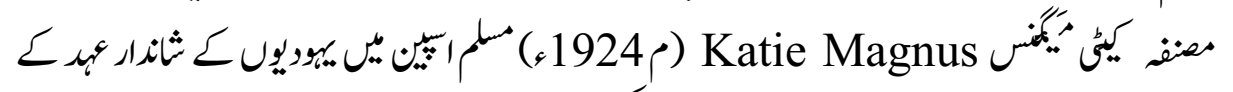

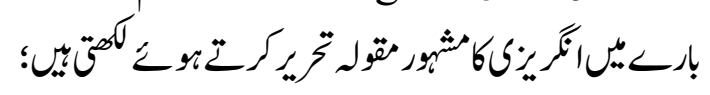

"Like a dream in the night - Life in Spain"36

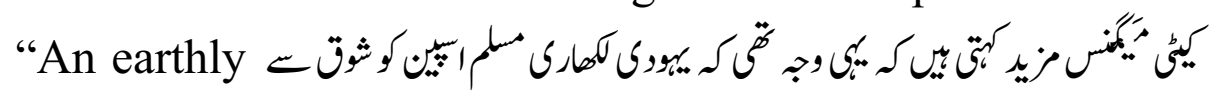

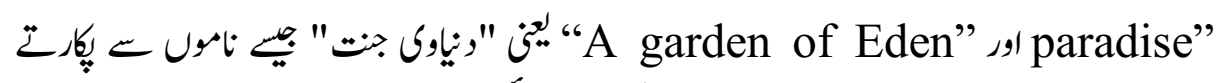

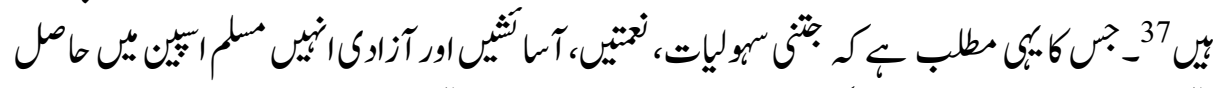

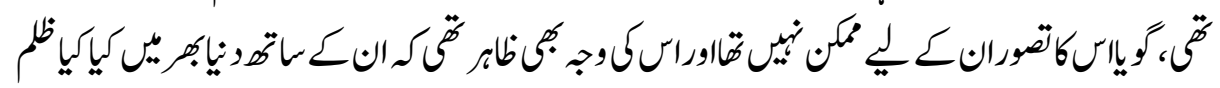

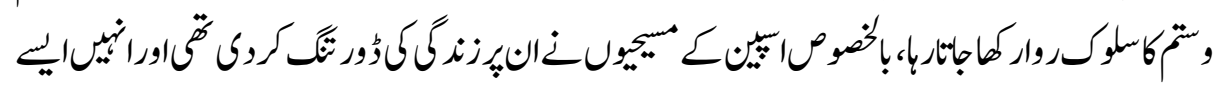

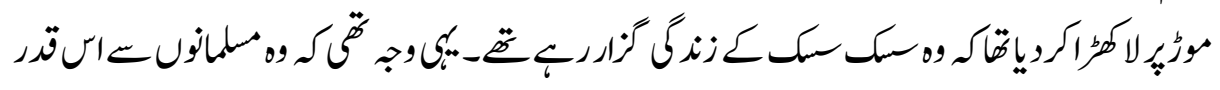

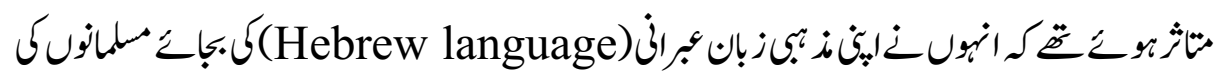

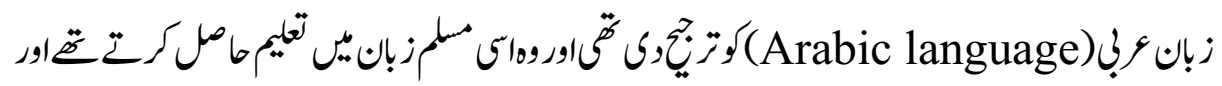

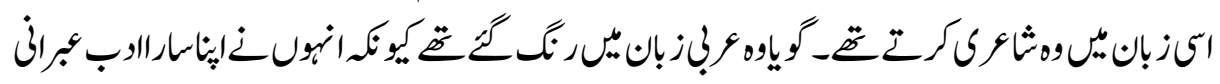




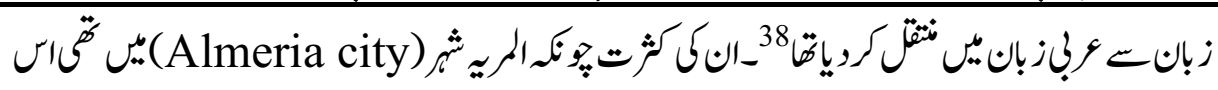

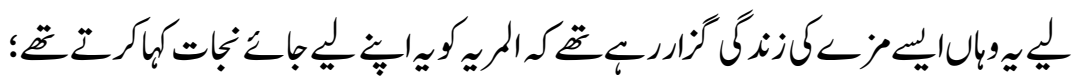

Almeria is a place of Salvation. ${ }^{39}$

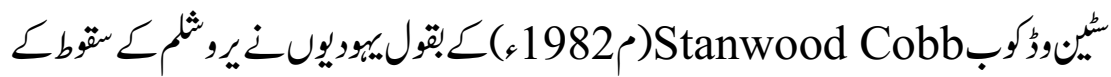

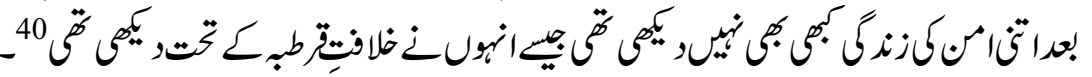

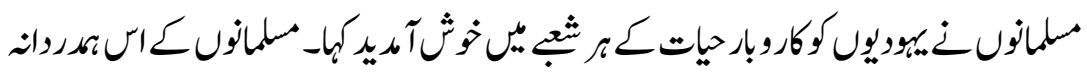

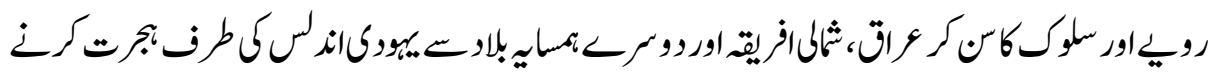

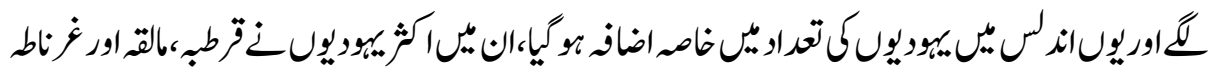

مي سكونت اضتياركى

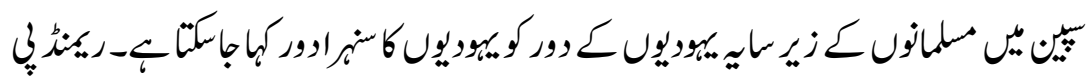

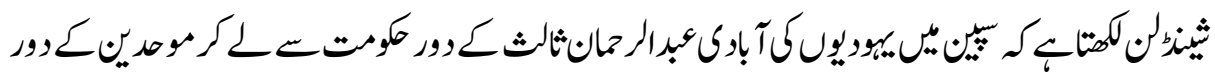

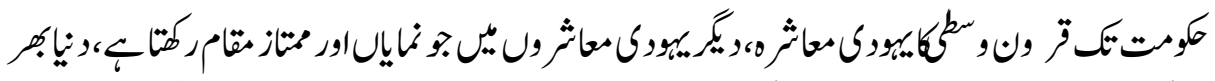

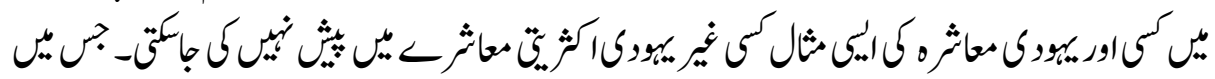

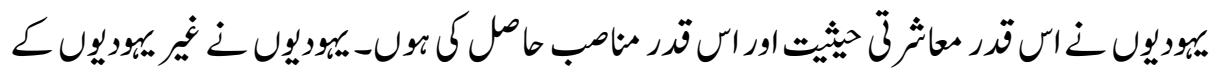

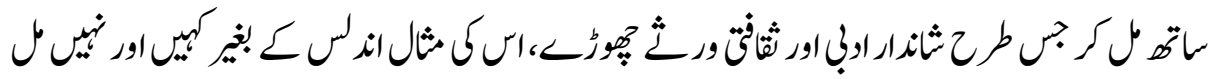

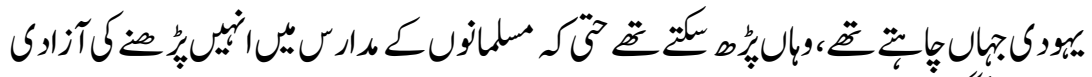

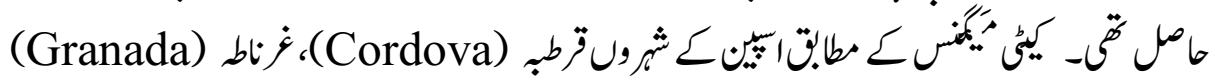

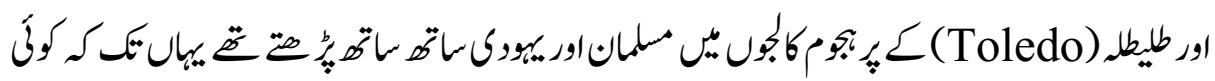

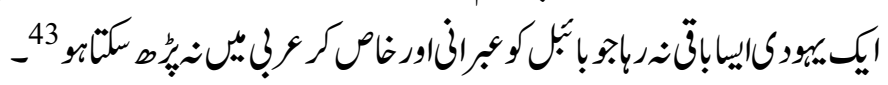

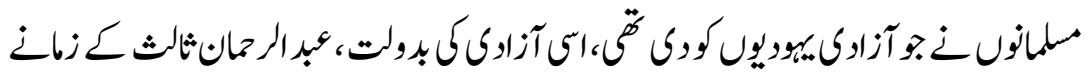

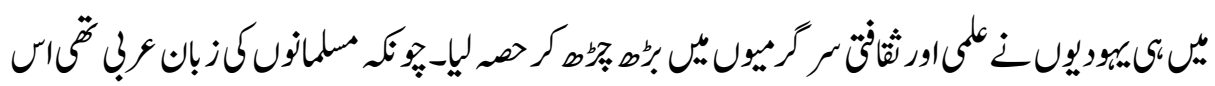

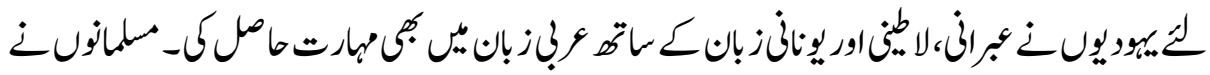

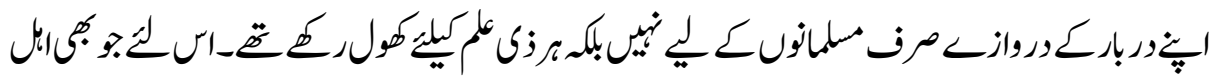




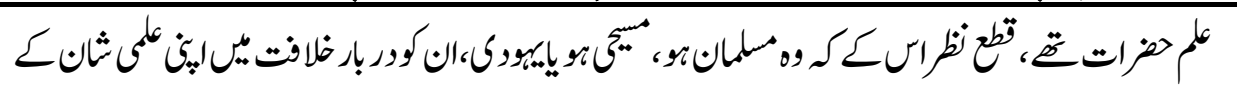

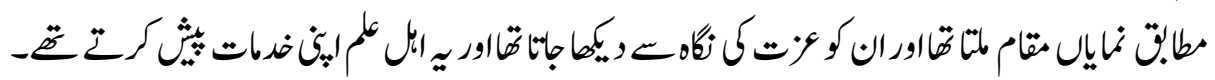

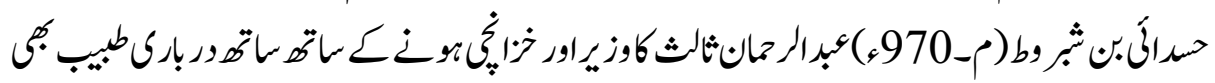

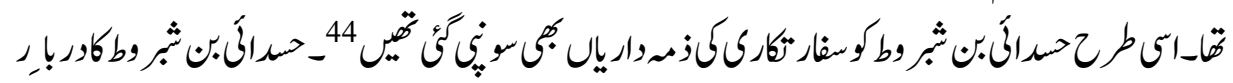

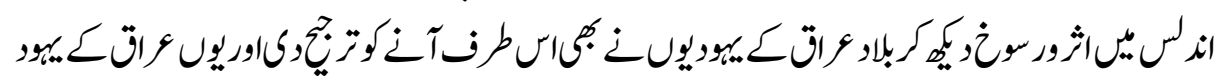

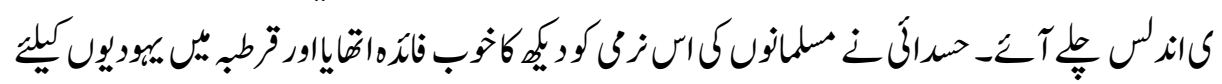

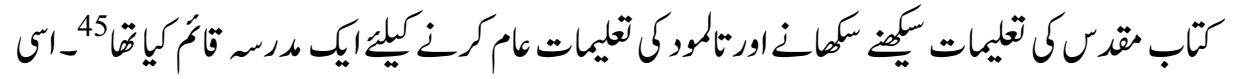

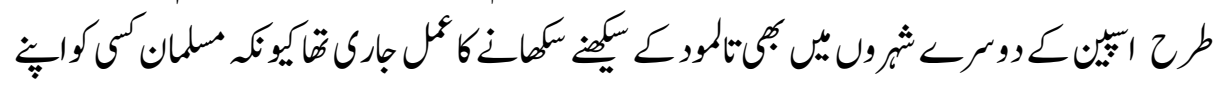

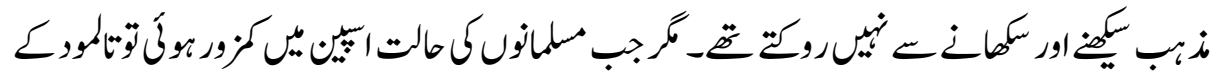

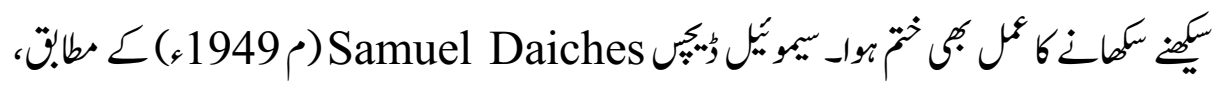

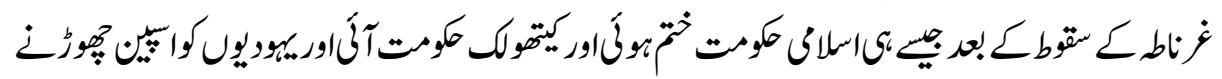

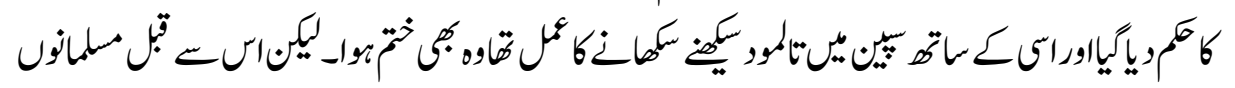

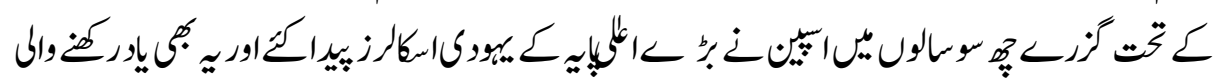

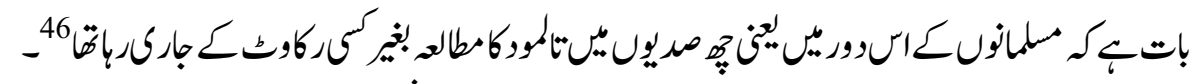

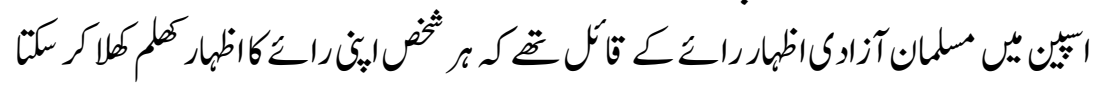

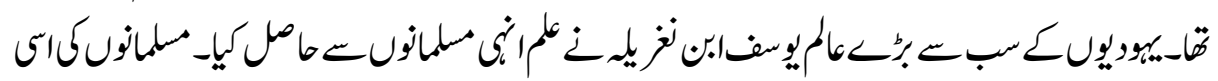

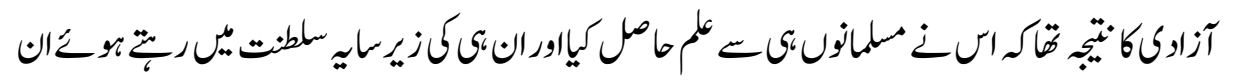

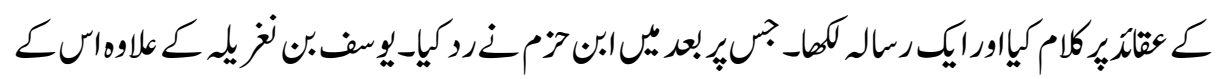

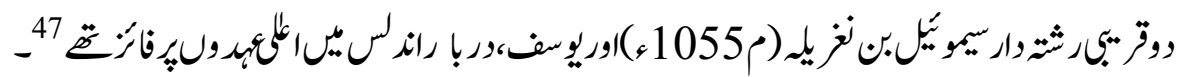

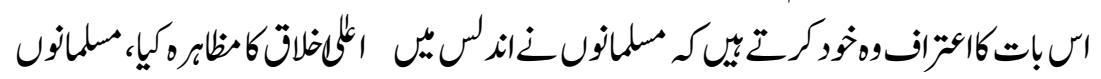

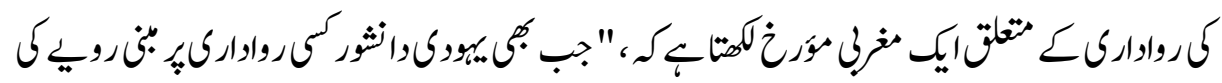

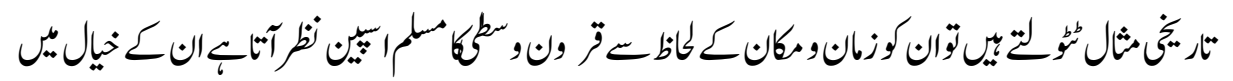

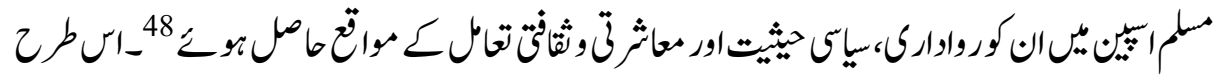




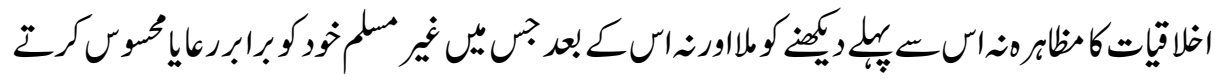

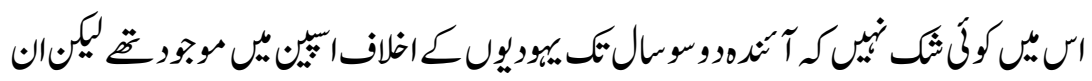

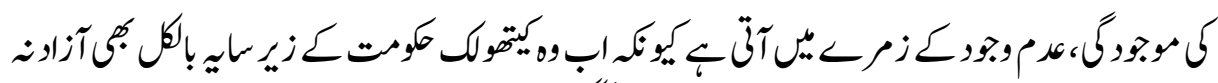

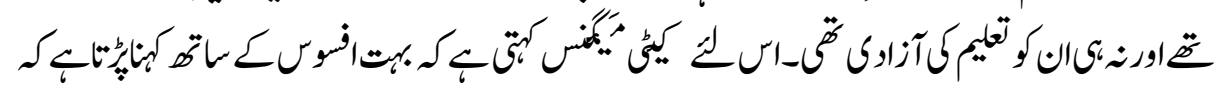

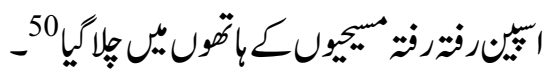

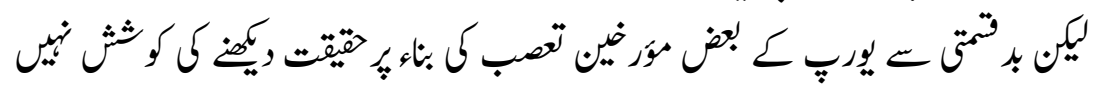

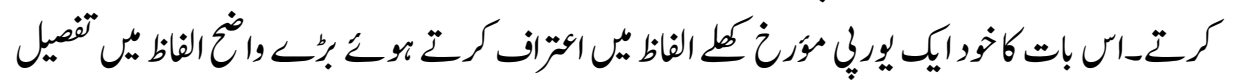
L

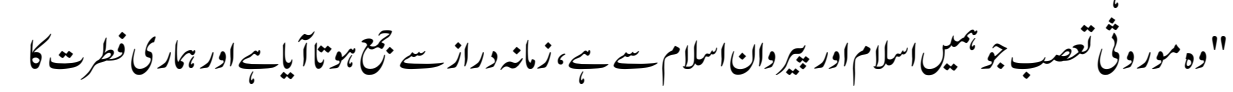

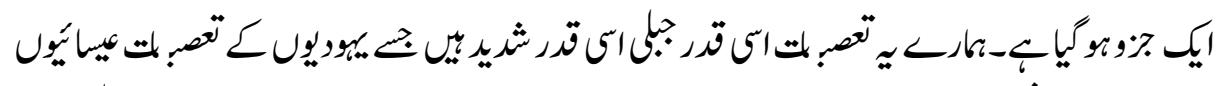

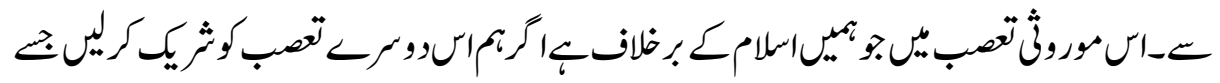

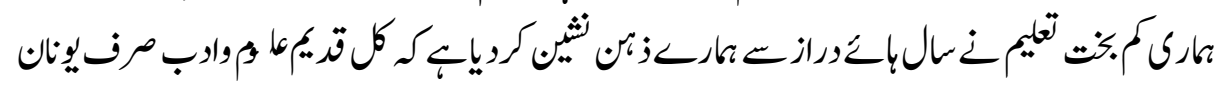

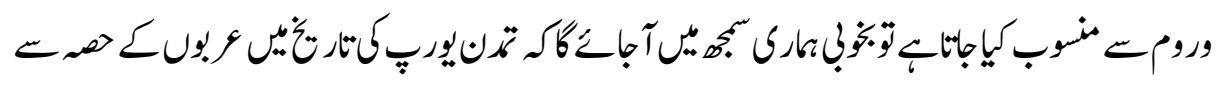

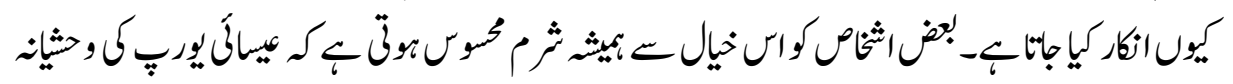

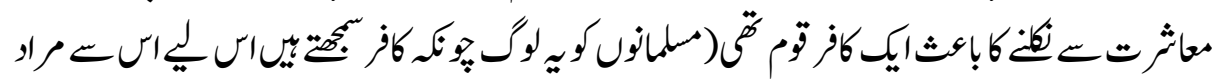

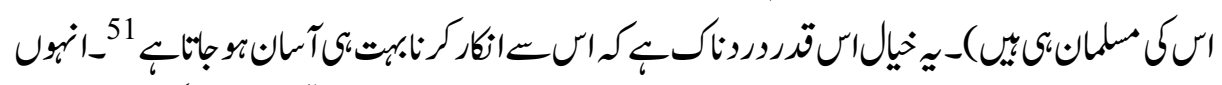

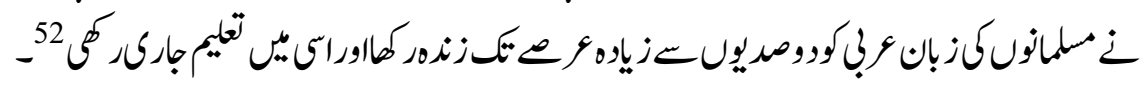

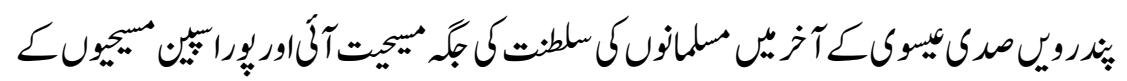

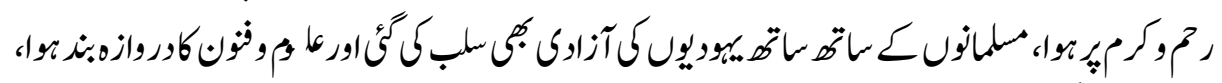

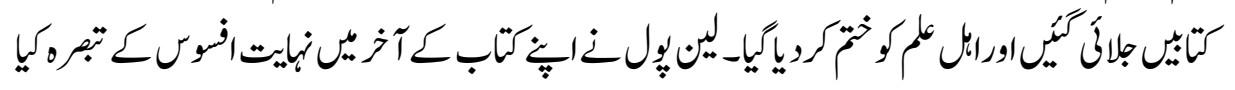
$c_{6} \log _{i} 09$ The Moors were banished, for a while the Christian Spain shone, like the Moon, with a borrowed light, then came the eclipse, and in that darkness Spain grovelled ever since $^{53}$ 


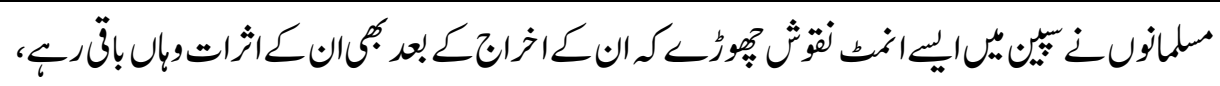

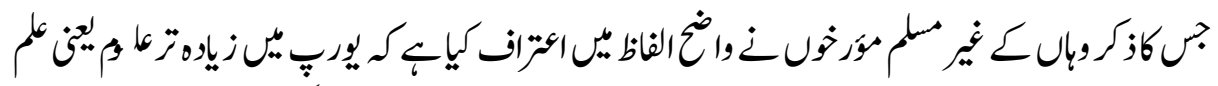

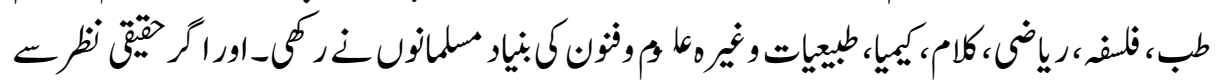

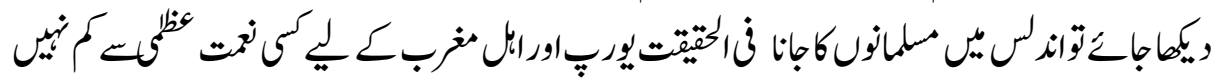

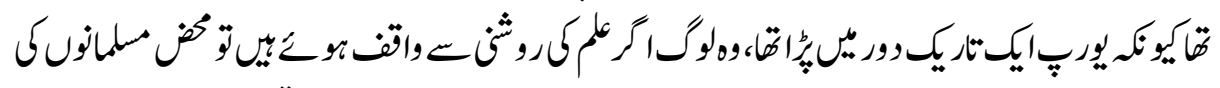

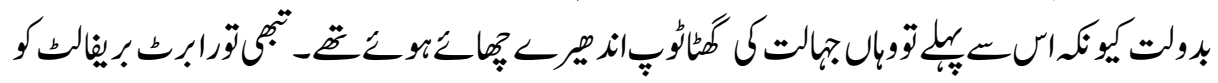

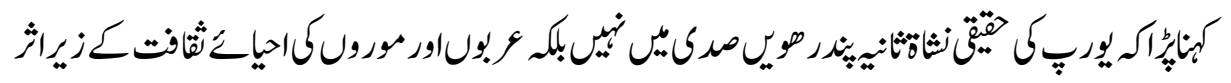

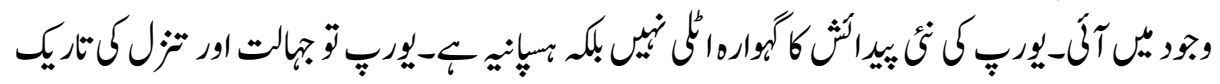

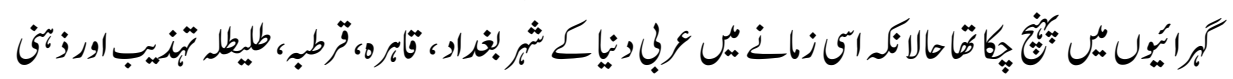

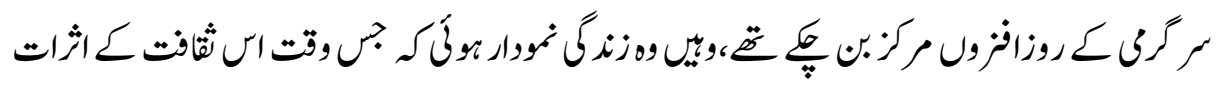

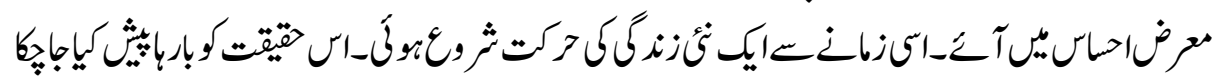

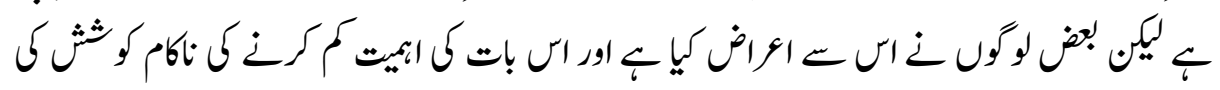

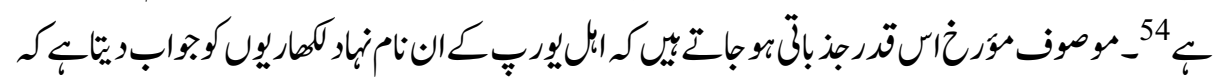

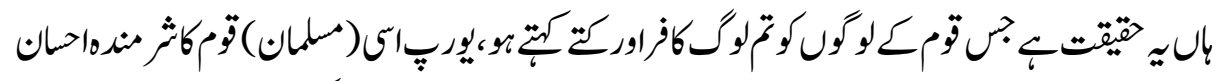

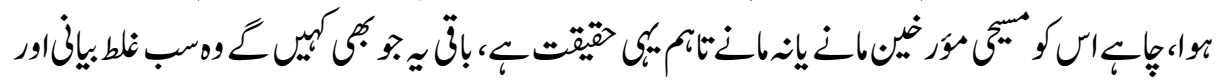

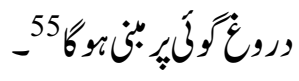 \\ رواراركـاثخات}

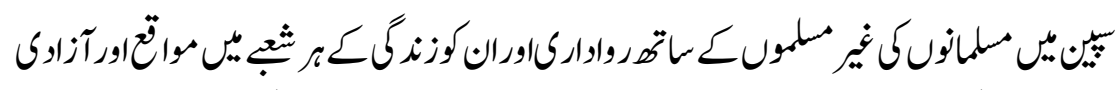

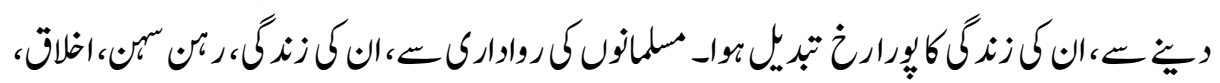

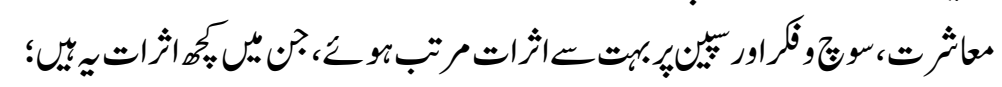

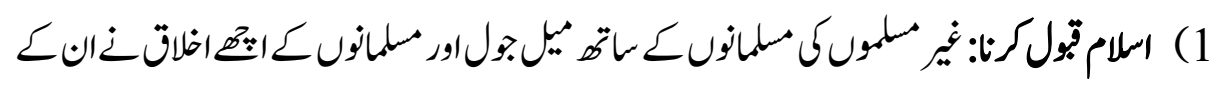

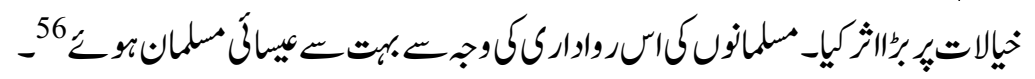

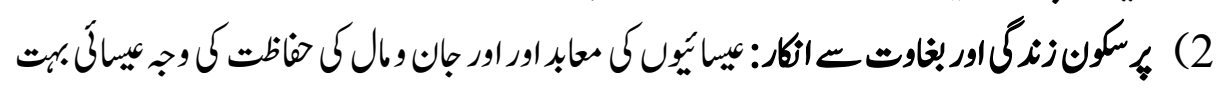

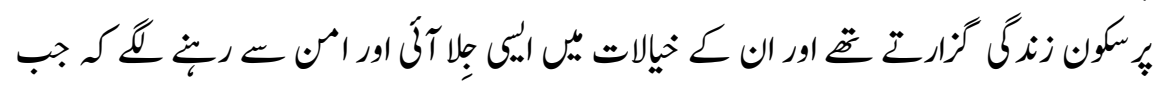




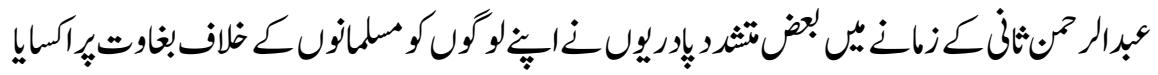

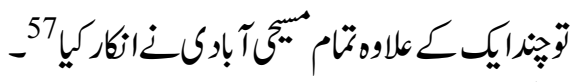

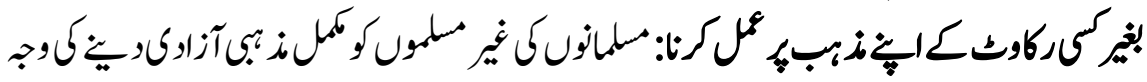

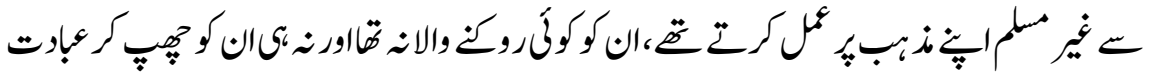

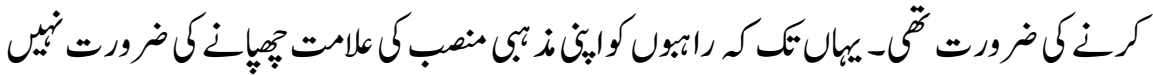

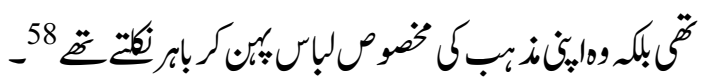

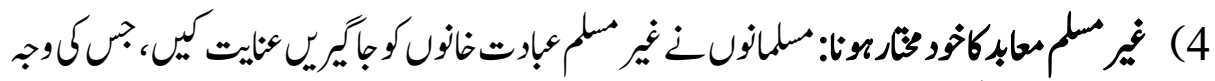

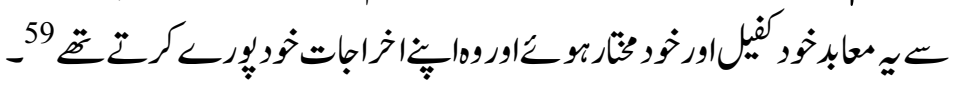

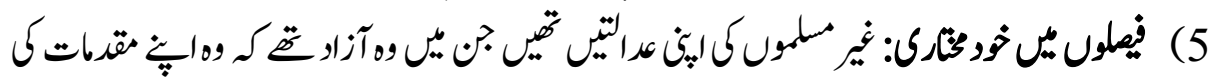

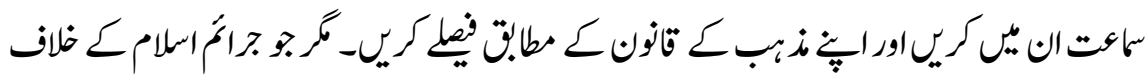

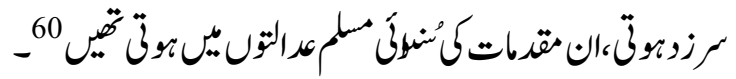

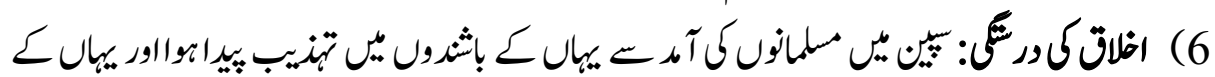

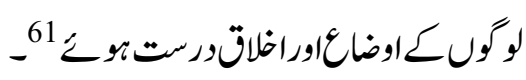

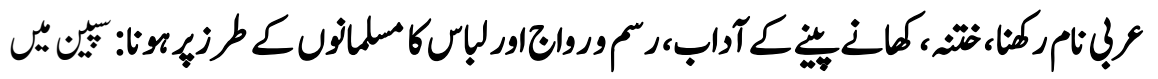

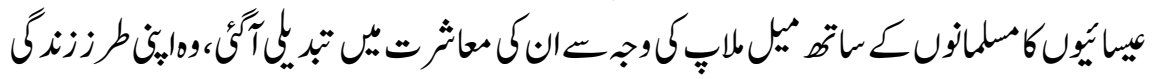

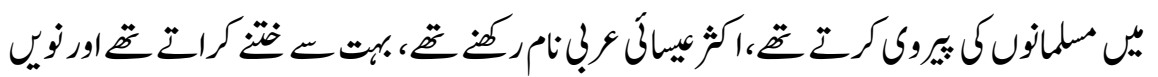

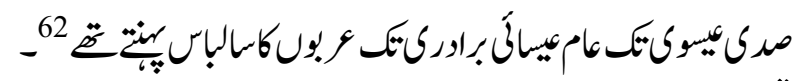

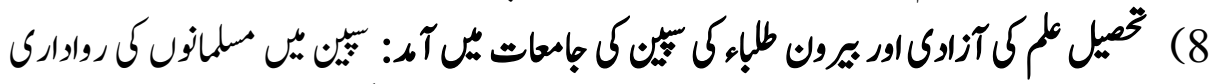

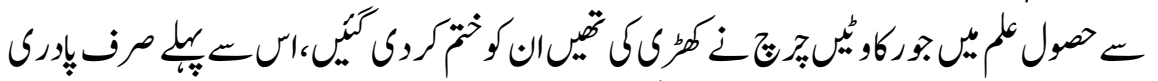

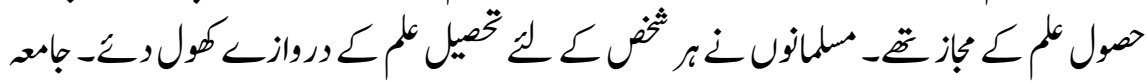

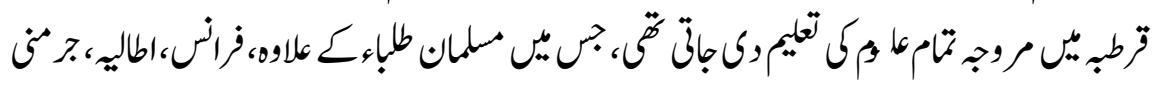

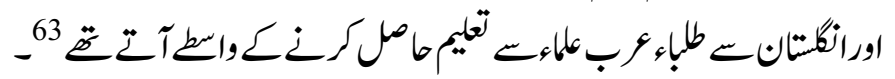




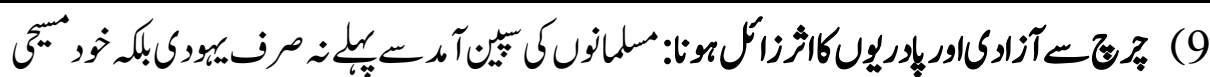

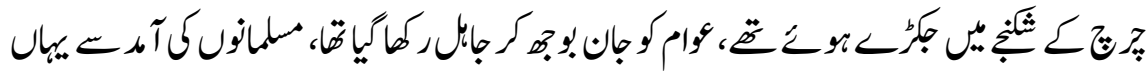

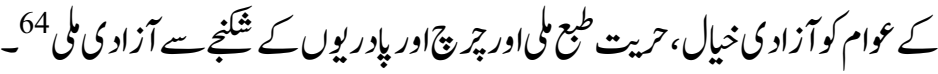
10

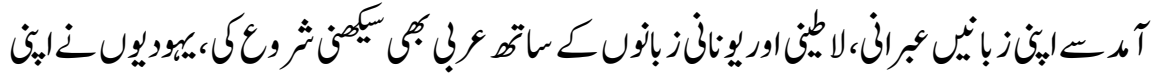

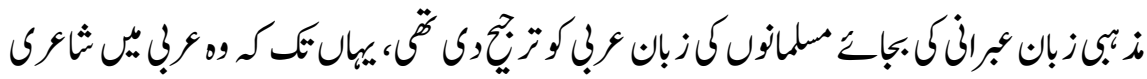

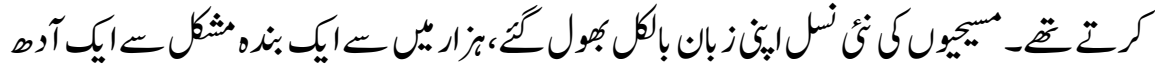

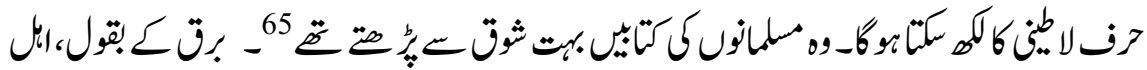

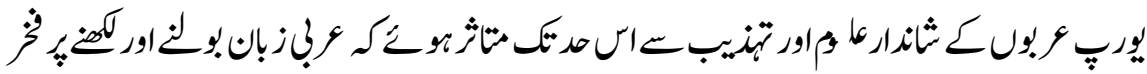
كركـ

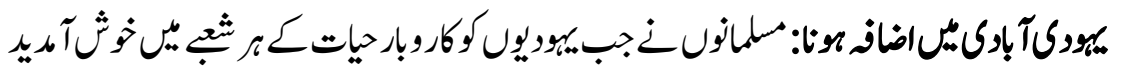

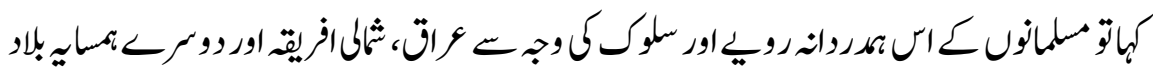

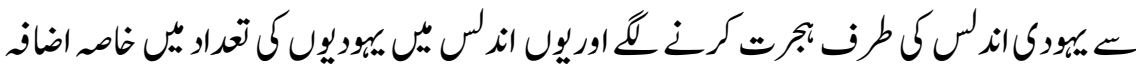

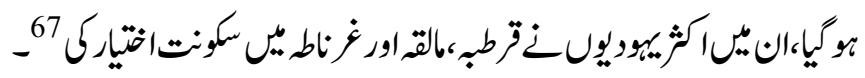

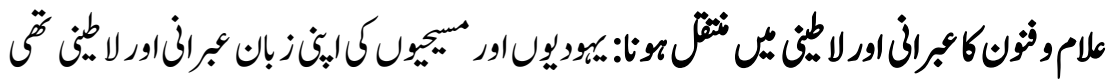

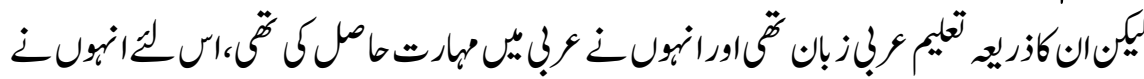

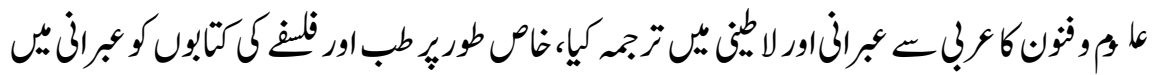

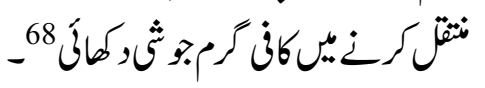

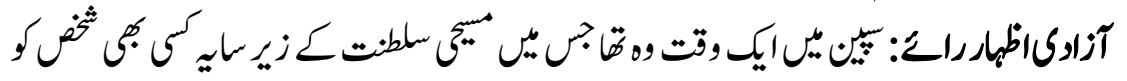

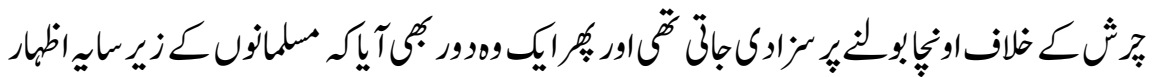

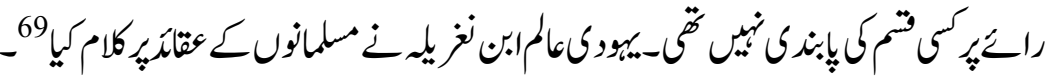

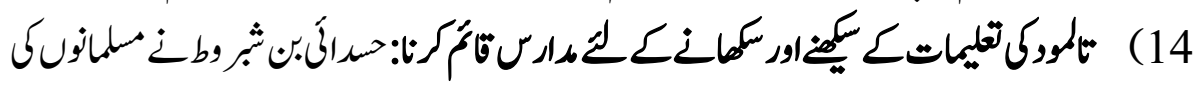

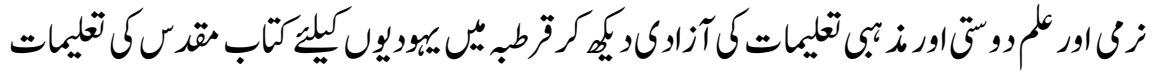

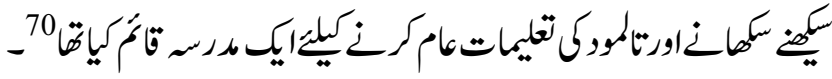




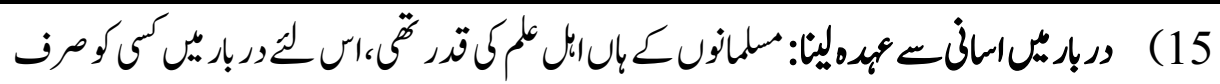

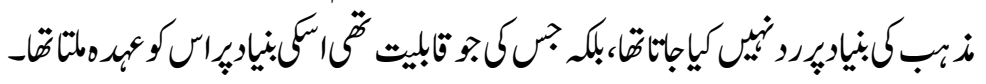

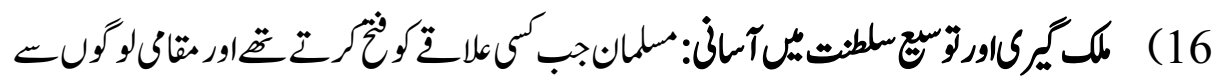

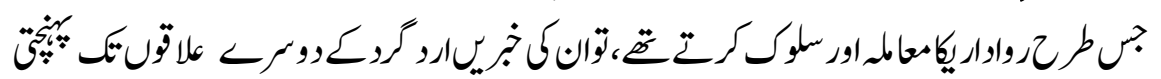

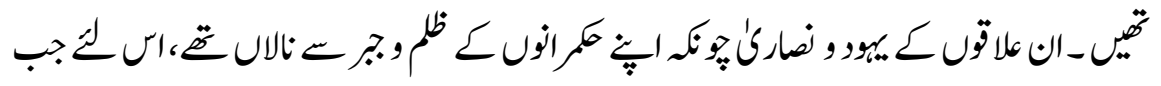

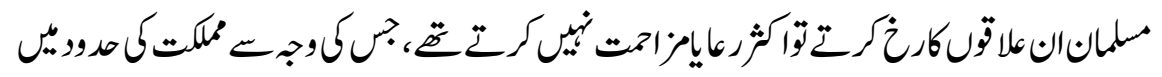

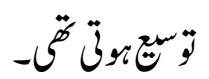

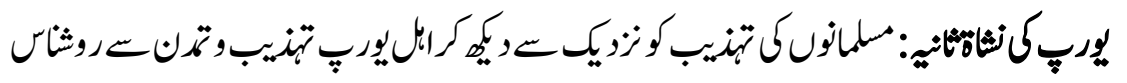

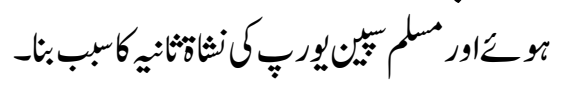

مارم

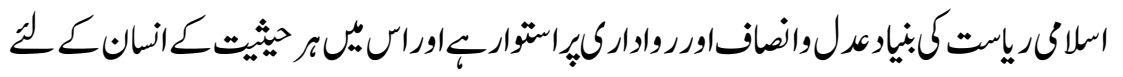

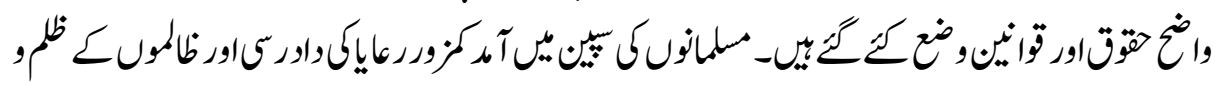

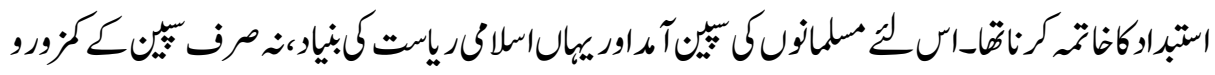

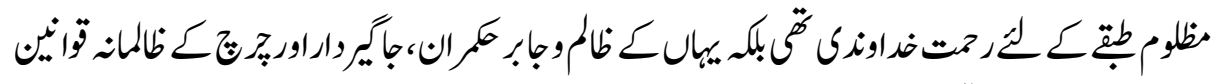

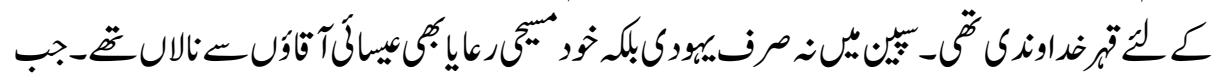

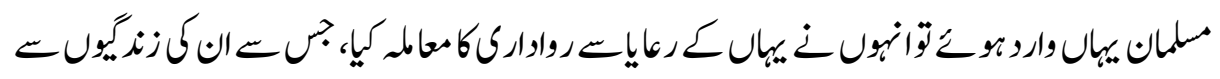

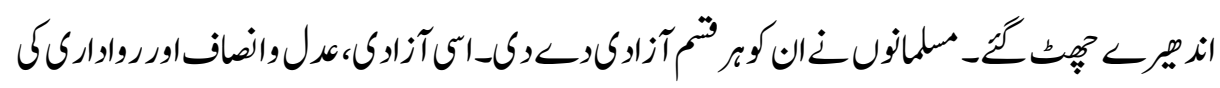

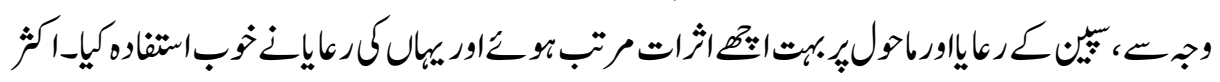

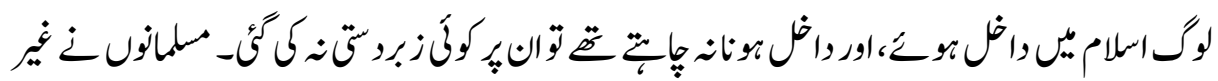

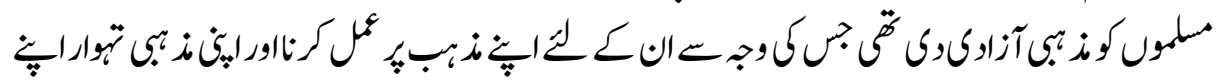

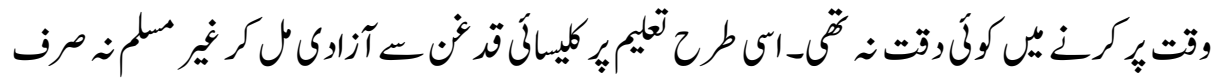

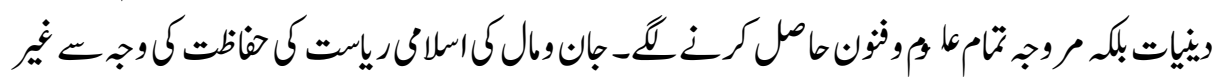

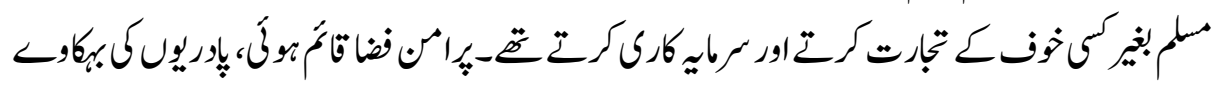



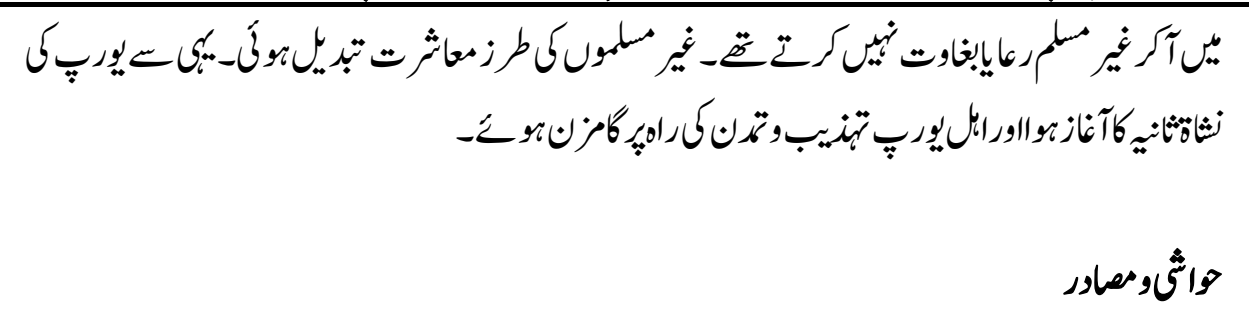

\section{References}

1. Al Baqara: 256

2. Al Younas: 99

3. Al Ana'am: 108

4. Suliman bin A'ashath, Abu Dawood, Sunan Abi Dawood, al maktaba al A'asriay, Beirut, $3 / 170$

5. Ibidi $3 / 83$

6. Al damishqi, Ismail bin Umer bin Kathir, al Bidaya wa al nihay, darul Fikar, Beirut, 1986, 7/39

7. Al bakri, Abdullah bin Abdul Aziz bin Muhammad, al masalik wa al mamalik, darul gharab al Islami, Beirut, 2/781

8. Taqi Usmani, Muft, dunia mere aage, maktaba ma'arif al Quran, Karachi, 2010, p16

9. Wikipedia, org/ accessed 13 May 2019

10. Al Katani Ali bin Muhammad al Muntasar billah,inbia'as al Islam fil undulas, darul kutub al ilmia, 1426h, p29

11. Donini, Guido, History of Goths, Vandals and Suevi, Brill, 1970, p3

12. H. V. Livermore, The Origins of Spain and Portugal, Cambridge University Press, 1971, pp86-88

13. Nadavi, Riyasat Ali, Tarikh e Undulas, Darul Shaoor, Lahore, 2013, pp16

14. Ib al Qutawia, Tarikh iftitah al undula,Kholian rabira, 1926, pp.38

15. www.searchtruth.com/hijri/ accessed 19 June 2019

16. Mehar, Ghulam Rasool, Mukhtasar Tarikh e Islam, Mushtaq book corner, Lahore, 2011, pp. 224

17. Hitti, Philip, History of the Arabs, Red Glob Publisher, New York, 2002, pp. 555 
18. Jang, zulqadar, khilafat e undulas, Mushtaq book corner, Lahore, 2001, pp.349

19. Nadavi, Riyasat Ali, Tarikh e Undulas, Darul Shaoor, Lahore, 2013, pp. 27

20. Ibid

21. Khalil Ibrahim al Samari, Tarikh al Arab wa hazarathim fil undulas, darul kutub al jaded, Beirut, 2001, pp. 16

22. Haq, Mazhar ur, A Short History of Muslims Spain, Book land publishers, Lahore, 1988 , pp. 6,7

23. Shahabi, intizamullah, salatin e undulas, maktaba Faridi, Karachi, 1956, pp. 13

24. Nadavi, Riyasat Ali, Tarikh e Undulas, Darul Shaoor, Lahore, 2013, pp. 28

25. Khan, Qudratullah, Islami haspania, 1978, pp. 63

26. Muhammad, Ahmad Syed, Tarikh e spain, Mushtaq book corner, Lahore, 2010, pp. 574

27. Goitein, Jes and Arabs, Schochen Book, New York, 1074, pp. 62

28. Muhammad, Ahmad Syed, Tarikh e spain, Mushtaq book corner, Lahore, 2010, pp. 576,577

29. Shahabi, intizamullah, salatin e undulas, maktaba Faridi, Karachi, 1956, pp. 13

30. Zulqadar, khilafat e undulas, pp. 376

31. Shahabi, intizamullah, salatin e undulas, maktaba Faridi, Karachi, 1956, pp. 91

32. Ibid, pp. 190

33. Lane- people, Stanly, The Story of the Moors in Spain, G. P. Putnam's sons, New York, 1898, pp. 90

34. Haq, Mazhar ur, A Short History of Muslims Spain, Book land publishers, Lahore, 1988, pp. 6,7

35. Goiteing, Jews and Arabs, pp. 62

36. Magnus, Katie, Outlines of Jewish History, Jewish Publications society, Philadephia, 1980, pp. 130

37. Ibid, pp. 130

38. Ashtor, The Jews of Moslem Spain, Jewish Publications society, Philadephia, 1973, pp. 388

39. Goiteing, Jews and Arabs, pp. 125 
40. Cobb, Stanwood, Islamic contribution to civilization, Avalon Press, New York, 1963, pp. 37

41. Muhammad Ahmad, Tarikh e Sapain, pp. 568

42. Jayyusi,Salma Khadra, The legacy of Muslim Spain, pp. 188

43. Magnus, Katie, Outlines of Jewish History, pp. 132

44. Jayyusi, Salma Khadra, The Legacy of Muslim Spain, pp. 192

45. Wiipedia. Org. accessed, 21 June 2019

46. Daiches, Samuel, The Study of the Talmud in Spain, R. Mazin \&co. 1td, 1921, pp. 29,30

47. Ashtor, The Jews of Moslem Spain, pp. 72

48. Cohen, Mark, R. Under Crescent and Cross: The Jews in the middle Ages, Princeton University Press, New Jersey, 1995, pp.3

49. Goitein, Jes and Arabs, pp. 125

50. Magnus, Katie, Outlines of Jewish Histroy, pp. 136

51. Gustawli ban, Tamaddan e Arab, al Faisal Nashiran, Lahore, pp. 330

52. Burns, Islam under the Crusader, University of Miami Press, 1974, pp. 238

53. Lane-poole, Stanley, The Story of the Moors in Spain, pp. 280

54. Brifalt, Robert, Tashkil e insaniat, majlis e taraqi e adab, 1994, pp. 254

55. Ibid

56. Shahabi, intizamullah, salatin e undulas, maktaba Faridi, Karachi, 1956, pp. 47

57. Zulqadar, khilafat e undulas, pp. 376

58. Muhammad Ahmad, Tarikh e Spain, pp. 577

59. Shahabi, intizamullah, salatin e undulas, maktaba Faridi, Karachi, 1956, pp. 190

60. Arnodl, T. W., The Preaching of Islam, Constable and company, London, 1913, pp. 135

61. Shahabi, intizamullah, salatin e undulas, maktaba Faridi, Karachi, 1956, pp. 51

62. Arnodl, T. W., The Preaching of Islam, Constable and company, London, 1913, pp. 135

63. Shahabi, intizamullah, salatin e undulas, maktaba Faridi, Karachi, 1956, pp. 91

64. Khan, Qudratullah, Islami Haspania, pp. 48

65. Lane-poole, Stanley, The Story of the Moors in Spain, pp. 90 
66. Burq, Ghulam Jilani, Erurope par Islam ke Ihsan, al Faisal Nashiran, Lahore, 2011, pp. 133

67. Muhammad Ahmad, Tarikh e Spain, pp. 568

68. Ibid, 572

69. Ashtor The Jews of Moslem Spain, pp. 72

70. Wikipedia.org accessed 21 June 2019 